\title{
Numerical Investigation of the Interaction of Counterflowing Jets and Supersonic Capsule Flows
}

\author{
Balaji Shankar Venkatachari*, Yasushi Ito ${ }^{\dagger}$, and Gary Cheng* \\ University of Alabama at Birmingham, Birmingham, AL, 35294 \\ and \\ Chau-Lyan Chang ${ }^{\S}$ \\ NASA Langley Research Center, Hampton, VA, 23681
}

\begin{abstract}
Use of counterflowing jets ejected into supersonic freestreams as a flow control concept to modify the external flowfield has gained renewed interest with regards to potential retropropulsion applications pertinent to entry, descent, and landing investigations. This study describes numerical computations of such a concept for a scaled wind-tunnel capsule model by employing the space-time conservation element solution element viscous flow solver with unstructured meshes. Both steadystate and time-accurate computations are performed for several configurations with different counterflowing jet Mach numbers. Axisymmetric computations exploring the effect of the jet flow rate and jet Mach number on the flow stability, jet interaction with the bow shock and its subsequent impact on the aerodynamic and aerothermal loads on the capsule body are carried out. Similar to previous experimental findings, both long and short penetration modes exist at a windtunnel Mach number of 3.48. It was found that both modes exhibit non-stationary behavior and the former is much more unstable than the latter. It was also found that the unstable long penetration mode only exists in a relatively small range of the jet mass flow rate. Solution-based mesh refinement procedures are used to improve solution accuracy and provide guidelines for a more effective mesh generation procedure for parametric studies. Details of the computed flowfields also serve as a means to broaden the knowledge base for future retropropulsion design studies.
\end{abstract}

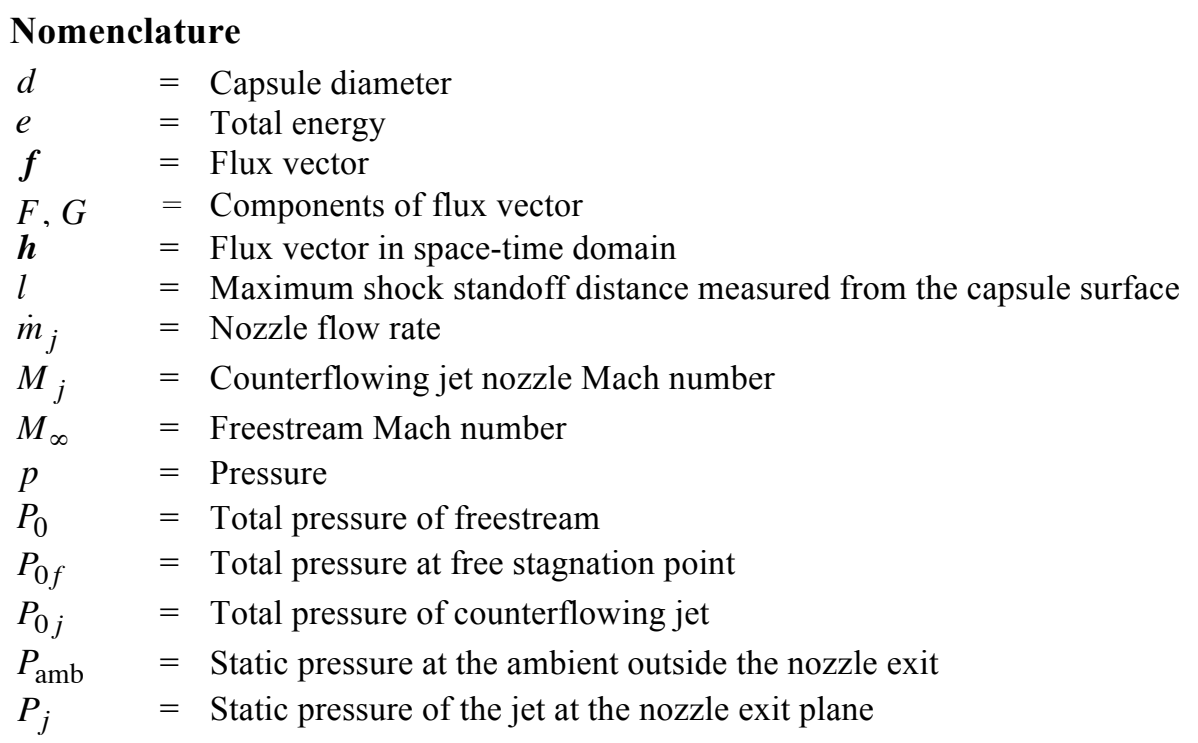

\footnotetext{
* Postdoctoral fellow, Department of Mechanical Engineering, email: balaji@uab.edu, Member AIAA.

${ }^{\dagger}$ Research Assistant Professor, Department of Mechanical Engineering, email: yito@,uab.edu, Senior Member AIAA.

* Associate Professor, Department of Mechanical Engineering, email: gcheng@uab.edu, Associate Fellow AIAA.

$\S$ Aerospace Technologist, Computational AeroSciences Branch, email: Chau-Lyan.Chang@nasa.gov, Senior Member AIAA.
} 


$\begin{array}{ll}P_{S} & =\text { Static pressure of freestream } \\ t & =\text { Time } \\ T_{0} & =\text { Total temperature of freestream } \\ T_{0 j} & =\text { Total temperature of counterflowing jet } \\ T_{S} & =\text { Static temperature of freestream } \\ u, v & =\text { Cartesian velocity components } \\ U & =\text { Conserved flow variable vector } \\ V & =\text { Space-time region } \\ x, y & =\text { Cartesian coordinates } \\ \gamma & =\text { Ratio of the specific heat } \\ \rho & =\text { Density }\end{array}$

\section{Background and Introduction}

Injection of a supersonic jet into an opposing supersonic/hypersonic freestream as a flow control concept to reduce aerothermal loads on spacecraft or as a decelerator technology during decent into planetary atmospheres like that of Mars, has recently gained renewed interest. ${ }^{1-3}$ Most studies on the use of opposing jets for such purposes were performed in the pre-Viking era. ${ }^{4-14}$ The initial studies were focused on the effects of retropropulsion nozzle flows on boundary-layer transition as well as the aerodynamic drag. ${ }^{4-6}$ The primary focus of these investigations were not on blunt-body geometries, but their results did lay the groundwork for understanding the physics involved in jet-shock interactions. The first of such studies on blunt bodies was by Stadler and Inouye ${ }^{7}$ in 1956, when they investigated the effect of air injection from the stagnation point on heat transfer for hemispherical bodies. Following their work, other studies such as those by Rashi ${ }^{8}$ and Ferri and Bloom ${ }^{9}$ also observed the surface cooling effects due to the injection of counterflowing water jets. Warren ${ }^{10}$ experimentally studied the effects of injecting gases into a Mach 5.8 free-stream and observed that the gaseous coolant effectively reduced the surface heat load when the injected jet did not disturb the freestream, a phenomenon observed at lower gas flow rates. Interestingly, the surface heat load increased in their experiments at larger gas flow rates due to the formation of a stagnation circle near the surface. A more detailed study (experimental as well as analytical) on the effects of jet Mach numbers, flow rates and thrust coefficient on the unsteadiness of the flow was done by Finley. ${ }^{11}$ Following this effort, Bushnell and Huffman ${ }^{12}$ investigated the influence of the jet penetration distance on the cooling effects, which was then also studied in a greater detail by Jarvinen and Adams ${ }^{13}$ by utilizing a conical aeroshell body fitted with a single nozzle jet. They were the first to report two regimes of jet penetration, namely the long penetration mode (LPM) and shortpenetration mode (SPM), along with details on how the pressure ratio between that of the jet and the freestream influenced the transition between the two modes. McGhee ${ }^{14}$ furthered the study on centrally located nozzles issuing supersonic jets by characterizing the flow in terms of the nature of jet expansion (over-expanded, fully-expanded, and under-expanded jets). Based on the nature of the jet expansion, $\mathrm{McGhee}^{14}$ identified the regimes of unsteady and steady flows. Simultaneously, there have also been other studies focusing on peripheral nozzle configurations that could be used for retropropulsion applications. More recent works in this regard have focused on the effects of weakly ionized non-equilibrium plasma jets on the wave drag and heat flux for hypersonic configurations. For a more thorough historic background and recent efforts on these concepts, the reader is referred to Refs. 1 and 16.

Most of the above studies were for simplified aeroshell geometries such as a sphere-cylinder or sphere-cone, and very few dealt with more realistic entry or re-entry vehicle configurations. Furthermore, a complete understanding of the complex physics involved in the interaction of opposing jets with a supersonic freestream has not been established. With these aspects in mind Daso et al. ${ }^{15}{ }^{16}$ recently performed experiments in conjunction with computational studies to investigate the interaction between a supersonic freestream and counterflowing jet and their subsequent impact on the aerodynamic and aerothermal characteristics of a $2.6 \%$ scale Apollo capsule model. The studies involved cold jets alone and were carried out in the trisonic wind tunnel of the Aerodynamic Research Facility at NASA Marshall Space Flight Center by employing two different freestream Mach numbers of 3.48 and 4.0. The effects of nozzle geometry, Mach number and mass flow rates of the counterflowing jets were investigated with a limited parametric space. In addition to Schlieren images, time-averaged pressure and heat flux data was also obtained as part of the study. Details on other available experimental databases can be found in Ref. 2 .

Parallel to the experimental work, computational studies are needed to help establish details of flow physics and expand the knowledge base for the effects of counterflowing jets. Given the nature of the intricate flow physics involved, predictive capabilities of currently available flow solvers must also be carefully assessed. Despite the fact that most solvers have been extensively used for simulations of complex flow problems, accurate computations of the counterflowing jet problem pose challenges due to the presence of potentially strong unsteadiness and complex shock-shock and shock-boundary layer interactions. In order to numerically resolve such complex flows with high-fidelity, we use the emerging space-time 
conservation element, solution element (CESE) method, ${ }^{17}$ a time-accurate numerical method for unstructured meshes (triangular meshes in 2-D and tetrahedral meshes in 3-D) designed to enforce strong flux conservation and capture unsteady waves and shocks simultaneously without ad-hoc numerical tuning.

To capture the physics faithfully and efficiently, the high-resolution, genuine multi-dimensional space-time CESE methodology solves the original integral form of the conservation laws rather than the differential form. The space-time CESE framework is unique for several reasons: (i) space and time are unified and treated as a single entity; (ii) local and global space-time flux conservation is enforced; (iii) no dimensional-splitting approach is used, resulting in a truly multidimensional scheme; (iv) both the conservative flow variable and its spatial derivatives are treated as dependent variables; (v) a space-time staggered stencil is used such that fluxes at the cell interfaces can be evaluated without solving the Riemann problem; (vi) schemes are built from non-dissipative (space-time inversion invariant) core schemes, allowing for efficient control of numerical dissipation; and (vii) it is naturally compatible with unstructured triangular and tetrahedral meshes. To date, the CESE based solvers have been utilized to solve a variety of problems including inviscid acoustic waves, traveling and interacting shocks, detonation waves, and cavitation flows. ${ }^{17-21}$

The main objective of this study is to perform numerical computations for selected experimental configurations studied by Daso et al. ${ }^{16}$ Axisymmetric computations are carried out to study the underlying physics associated with the two modes of jet interaction. Results are compared against available experimental data, such as Schlieren images, and other details available in literature. Similar studies using the original CESE schemes ${ }^{17,22}$ were performed for selected configurations with short penetration mode in Ref. 23. The main focus of the present study is to perform more detailed time-accurate computations using the updated CESE schemes ${ }^{24}$ for unsteady cases observed in the experiments in an attempt to explore the unsteady dynamics of the process. The most important update to the CESE scheme, relevant to this study, deals with the procedure to compute the flow variable derivatives - one of the dependent variables in the CESE methods. The new procedure $^{24}$ to compute derivatives provides the CESE method with the ability to effectively handle highly stretched unstructured meshes and the ability to deal with very strong shocks. As part of the study, the effect of the jet mass flow rates and the transition from the unstable LPM into the more stable SPM will be investigated to gain a deeper understanding of the retropropulsion concept. As flow instability plays a crucial role for drag reduction, such studies may significantly enhance design confidence for potential retropropulsion applications. Another aspect of this study is to explore the use of a solution-based mesh refinement method to better resolve important flow features. Given the complex nature of the flow involved in the various flow conditions targeted, it would be difficult to generate an ideal mesh a-priori unless a highly refined mesh is used. Results obtained in this study augment on-going computational studies of similar retropropulsion concepts using other well-established flow solvers. ${ }^{2,3}$ In the next section, we briefly describe the flow characteristics of a counterflowing jet in Section II, followed by the numerical method in Section III. Computational results and adaptive grid refinement studies are presented in Section IV. Conclusions from this study are summarized in Section V.

\section{General Flow Characteristics}

Supersonic flows around blunt-body geometries are in general characterized by a detached bow shock. The introduction of a supersonic jet (one that opposes the freestream) at or near the stagnation point of the blunt body can significantly alter the flowfield around the body. This modification to the flowfield is brought by the interaction between the jet flow and the bow shock, and is found to vary based on the location of the jet and its intensity. In this section we primarily focus on the central nozzle configuration and briefly describe the associated flow physics because of its relevance to cases studied by Daso et al. ${ }^{16}$

As detailed by Finley ${ }^{11}$, the primary flowfield characteristics of a counterflowing jet ejected from a central nozzle configuration are a bow shock, a jet terminal shock, free stagnation point and recirculation regions, as indicated in Fig. 1 (a). Based on the ratios of the total pressure of the jet and the freestream, features and the stability of the flowfield vary. The overall structure of the complex jet-bow shock interaction and the surrounding flowfield is primarily dependent upon the location of the free stagnation point, where both the freestream as well as the jet decelerate to zero velocity. For a capsule without counterflowing jets, the flow field configuration is nearly stable. However, for the central nozzle configuration shown in Fig.1, as the jet mass flow rate increases, the flow begins to become unstable and turns into a configuration with a diamond-pattern in the plume as given in Fig. 1(b). However, beyond some threshold value of the jet mass flow rate, the unstable flow reverts back into a near stable flow field with the structure shown in Fig. 1(a). The stable flowfield is characterized by a bow shock that remains close to the blunt body and the jet does not penetrate the bow shock. When the jet issued from the nozzle penetrates the bow shock, the shock displacement is significantly higher than that found in a stable flowfield (for the case with and without jet). In this scenario, the jet flow is unable to remain contained within the shock layer. Instead it begins to interact strongly with the bow shock resulting in a large shock standoff distance and creating an unstable flowfield with a very dispersed shock. As the jet flow rate increases further, for reasons not yet clearly understood, the shock standoff distance begins to decrease again and the flow eventually reverts back to a more stable condition, where the flowfield structure is characterized by a jet terminal shock and other flow features shown in Fig.1 (a). 
Jarvin and Adams ${ }^{13}$ characterized these two distinct flow regimes, where the jet does and does not interact with the bow shock as LPM and SPM respectively. Both modes are investigated computationally in this study.

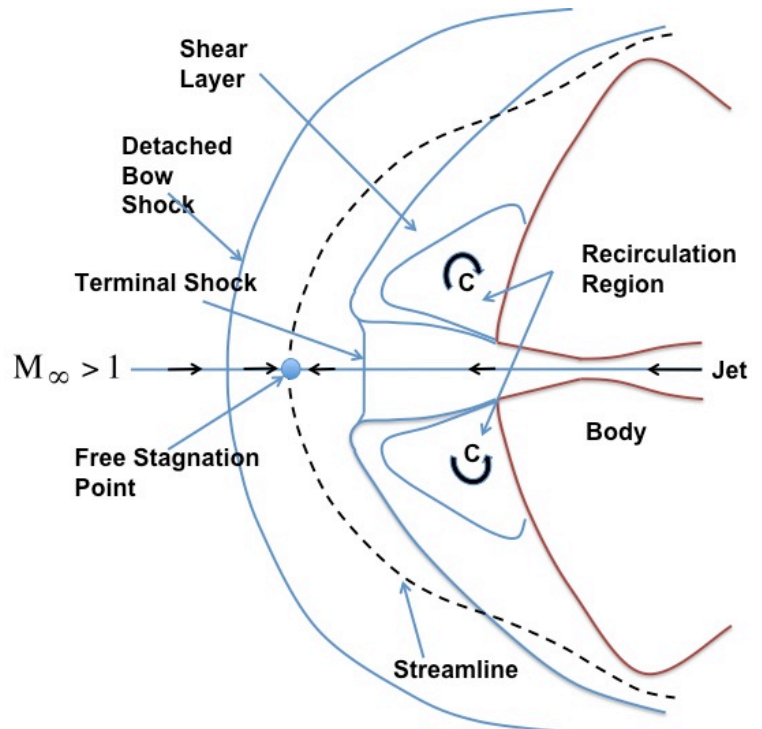

(a) Short Penetration Mode: Features of the stable flowfield for a central nozzle configuration

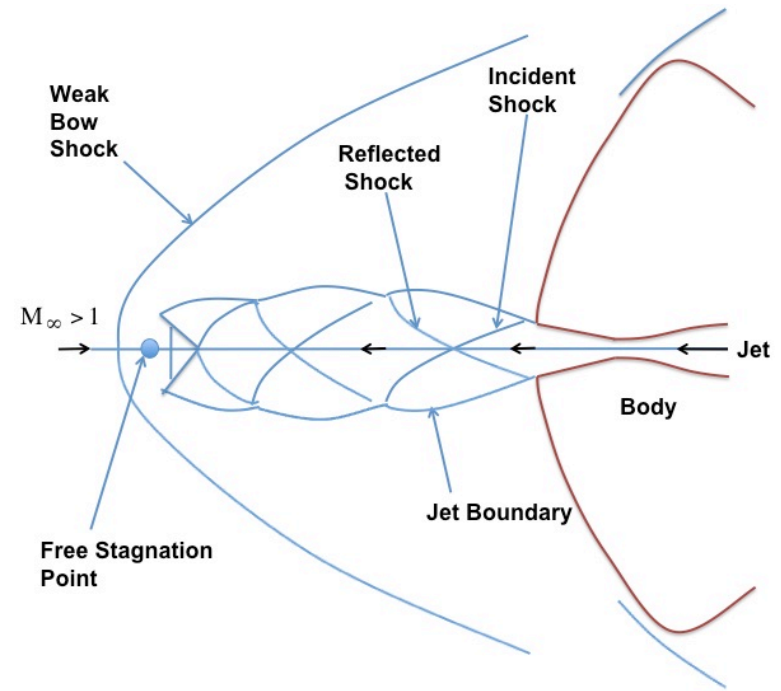

(b) Long Penetration Mode: Features of an unstable flowfield for a central nozzle configuration

Fig. 1. Flowfield structure characterization for a jet issuing from a central nozzle against a supersonic freestream.

\section{Space-Time CESE Method}

As mentioned in the Introduction Section, the space-time CESE method ${ }^{17}$ is built around the concept of treating space and time synergistically and conserving fluxes in both space and time. To bring out the clear conceptual difference between conventional CFD algorithms and the CESE framework, consider the differential form of a conservation law in multiple spatial dimensions that is given by

$$
\frac{\partial U}{\partial t}+\nabla \cdot f(U)=0
$$

where $U$ is the conserved variable, $f(U)$ is its spatial flux vector, and the symbol $\nabla \cdot$ denotes the divergence operator in the spatial domain. Finite-difference schemes model only the differential form of Eq. (1). Note that a set of physical conservation laws, in their original form, is a collection of flux conservation equations in space-time. These conservation laws are represented by a set of integral equations. The differential form of these laws is obtained from the integral form 
using an extra assumption that the physical solution is smooth. Consequently, by modeling only the differential form, finitedifference schemes, in general, lack the capacity to capture solution discontinuities and to ensure flux conservation. In an attempt to capture physics more accurately, conventional finite-volume schemes model the flux-balance form of Eq. (1) over a fixed spatial domain, i.e.,

$$
\frac{d}{d t} \int_{V} U d V=-\int_{S(V)} f(U) \cdot d s
$$

where $S(V)$ is the boundary of a spatial control volume $V$, and directional elemental area $d \boldsymbol{s}=d \sigma \boldsymbol{n}$ with $d \sigma$ representing the area and $\boldsymbol{n}$ the outward unit normal vector of a surface element on $S(V)$. Eq. (2) essentially states that the rate of increase of the total amount of a conservative substance contained in a fixed spatial domain $V$ is equal to the net flux of that substance entering the boundary of $V$. Finite-volume methods concentrate on evaluation of the right hand side of Eq. (2), and the left hand side of Eq. (2) is usually discretized by finite-differencing, such as the Runge-Kutta method. Thus, there is a clear distinction in the way the temporal and spatial derivatives of Eq. (2) are treated in a conventional finite-volume scheme.

Consider a special case of Eq. (1) with two spatial dimensions. Let $x$ - and $y$ be the spatial coordinates and $F$ and $G$ be the flux components of the flux vector $\boldsymbol{f}(U)$ in the $\mathrm{x}$ - and y-directions, respectively. Let $(x, y, t)$ be the coordinates of a three-dimensional Euclidean space, $\mathrm{E}_{3}$, shown in Fig. 2, and let the symbol $\underline{\nabla}$. denote the divergence operator in the space-time domain $E_{3}$. Then, Eq. (1) reduces to

$$
\underline{\nabla} \cdot \boldsymbol{h} \equiv \frac{\partial U}{\partial t}+\frac{\partial F}{\partial x}+\frac{\partial G}{\partial y}=0
$$

where $\boldsymbol{h}=(F, G, U)$ is the space-time flux vector. If the Navier-Stokes equations are solved as the governing equations with perfect gas flow assumption, then $U$ is defined as $U=(\rho, \rho u, \rho v, e)$, where the flow variables $\rho, u, v$, and $e$ represent density, two velocity components, and total energy (defined as $e=p /(\gamma-1)+\rho\left(u^{2}+v^{2}\right) / 2$, where $\mathrm{p}$ denotes the pressure), respectively. The flux vectors $F$ and $G$ include both the inviscid (Euler) and the viscous parts. Invoking Gauss's divergence theorem in $\mathrm{E}_{3}$, Eq. (3) can be expressed in the following integral form:

$$
\oint_{S(V)} \boldsymbol{h} \cdot d \boldsymbol{s}=0
$$

$S(V)$ is the boundary of an arbitrary space-time region $V$ (see Fig. 2) in $\mathrm{E}_{3}$, and and $d \boldsymbol{s}=d \sigma \boldsymbol{n}$ with $d \sigma$ and $\boldsymbol{n}$, respectively, denoting the area and the outward unit normal vector of a surface element on $S(V)$. Because $\boldsymbol{h} \cdot \boldsymbol{d} \boldsymbol{s}$ is the space-time flux of $\boldsymbol{h}$ leaving the space-time region $V$ through the surface element $d \boldsymbol{s}$, Eq. (4) simply states that the total space-time flux of $\boldsymbol{h}$ leaving $V$ through its boundary vanishes, i.e., the rigorous statement of the space-time flux conservation law associated with Eq. (3). Unlike Eq. (2), the form presented in Eq. (4) treats both the temporal and spatial terms of Eq. (1) in a unified manner. This is a crucial difference that, at the conceptual level, separates CESE schemes from the conventional finitevolume schemes. There are several schemes under the CESE framework; but those of relevance to the study here are the solution point based Courant number insensitive scheme ${ }^{22,25}$ and the edge-based Courant number insensitive scheme. ${ }^{24}$ These two schemes are vital for viscous simulations that involve a large disparity in the cell sizes across the mesh employed. Numerical dissipation control for cells with a very small Courant number plays a crucial role in numerical accuracy for such meshes. The edge-based method $^{24}$ is a relatively newer development and will be employed in all the computations performed in this study. It is invaluable to problems where the cell sizes vary by several orders across the mesh and have highly skewed shapes, as is the norm in many hypersonic computations. More details on these schemes can be obtained from Refs. 24 and 26. With its capability to compute supersonic viscous flows over a blunt body without any undesired numerical issues such as the carbuncle phenomenon, ${ }^{27}$ the CESE numerical framework provides a distinct advantage over other conventional numerical schemes. 


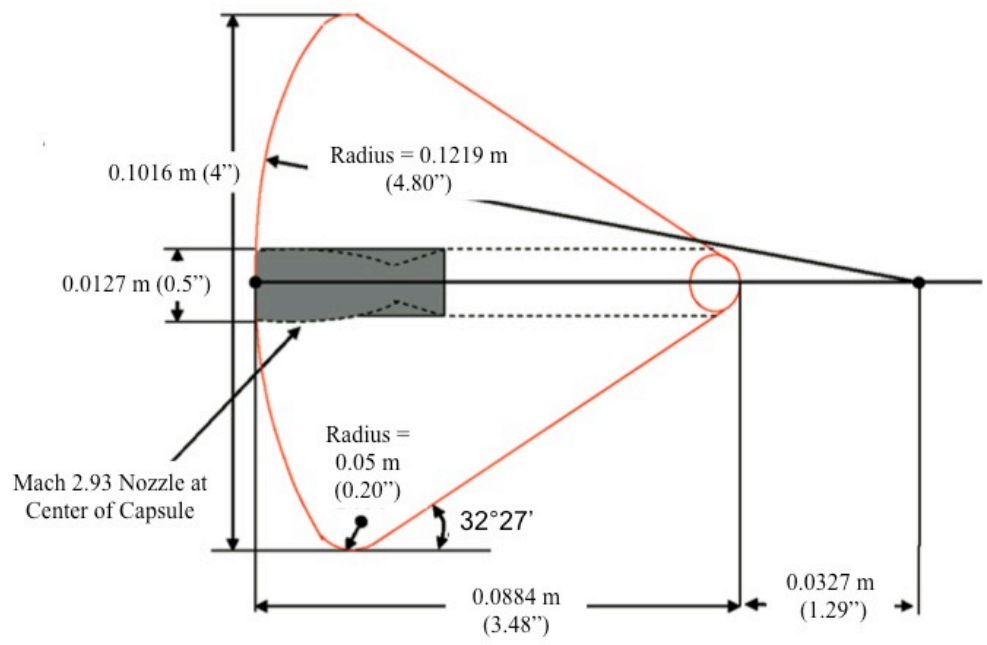

Fig 3. Experimental capsule model geometry (2.6\% subscale Apollo model) from Daso et al. ${ }^{16}$

Table 1. Freestream and counterfowing jet conditions simulated in the present study.

\begin{tabular}{|c|c|c|c|c|}
\hline \multicolumn{5}{|c|}{ 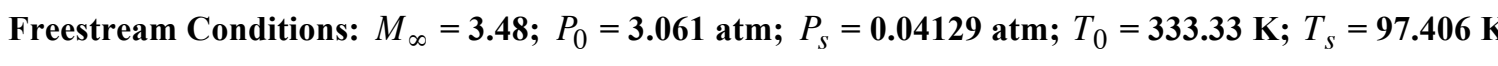 } \\
\hline \multicolumn{5}{|c|}{ Nozzle Conditions } \\
\hline$M_{j}$ & Case ID & $\dot{m}_{j}(\mathrm{~kg} / \mathrm{s})$ & $P_{0 j}(\mathrm{~atm})$ & $T_{0 j}(\mathrm{~K})$ \\
\hline 0 & Baseline & 0 & - & - \\
\hline \multirow{5}{*}{2.44} & $\mathrm{~N} 1 \mathrm{mf1}$ & $0.0227(0.05 \mathrm{lbm} / \mathrm{s})$ & 1.633 & 300.00 \\
\hline & $\mathrm{N} 1 \mathrm{mf} 2$ & $0.0453(0.1 \mathrm{lbm} / \mathrm{s})$ & 5.178 & 300.00 \\
\hline & $\mathrm{N} 1 \mathrm{mf3}$ & $0.0680(0.15 \mathrm{lbm} / \mathrm{s})$ & 5.444 & 300.00 \\
\hline & N1mf4 & $0.0907(0.2 \mathrm{lbm} / \mathrm{s})$ & 6.532 & 300.00 \\
\hline & $\mathrm{N} 1 \mathrm{mf5}$ & $0.2268(0.5 \mathrm{lbm} / \mathrm{s})$ & 19.441 & 300.00 \\
\hline \multirow{5}{*}{2.94} & $\mathrm{~N} 2 \mathrm{mf} 1$ & $0.0227(0.05 \mathrm{lbm} / \mathrm{s})$ & 2.966 & 300.00 \\
\hline & $\mathrm{N} 2 \mathrm{mf} 2$ & $0.0453(0.1 \mathrm{lbm} / \mathrm{s})$ & 6.294 & 300.00 \\
\hline & $\mathrm{N} 2 \mathrm{mf} 3$ & $0.0680(0.15 \mathrm{lbm} / \mathrm{s})$ & 9.241 & 300.00 \\
\hline & $\mathrm{N} 2 \mathrm{mf} 4$ & $0.0907(0.2 \mathrm{lbm} / \mathrm{s})$ & 12.207 & 300.00 \\
\hline & $\mathrm{N} 2 \mathrm{mf5}$ & $0.2268(0.5 \mathrm{lbm} / \mathrm{s})$ & 28.865 & 300.00 \\
\hline
\end{tabular}

\section{Results and Discussion}

Altough Daso et al. ${ }^{16}$ investigated the effect of a counterflowing jet on flow augmentation for three different angles of attack $\left(0^{\circ}, \pm 9^{\circ}\right)$, only the zero angle of attack case is investigated in this work. While Daso et al. ${ }^{16}$ had utilized five different nozzle geometries in their study only two of them (jet Mach numbers of 2.44 and 2.94) are studied in detail here. These two configurations are selected to represent the long and short penetration modes (LPM and SPM) observed in Daso et al.'s experiments. ${ }^{16}$ Additionally, flow without a counterflowing jet is also computed as the baseline solution. The geometry of the capsule tested is shown in Fig. 3 and the flow conditions for the various computations performed are summarized in Table 1. In Table 1, the various runs are designated with a unique case name. The first part of the case name, namely N1 or N2 stands for the nozzle chosen, with N1 and N2 representing the jet Mach number of 2.44 and 2.98, respectively. The second half (mf1-mf5) corresponds to which of the five jet mass flow rates is being computed. Since Daso et al. ${ }^{16}$ had listed the jet mass flow rates in English units, both SI and English units are listed here in Table 1 for reference. For all of the 
computations presented the freestream Mach number is 3.48, with a unit Reynolds number of $1.527 \times 10^{7} \mathrm{~m}^{-1}$ and the capsule and sting wall temperature of $297 \mathrm{~K}$.

Since the current study focuses only on zero angle of attack, the axisymmetric version of the Navier-Stokes equations is used for the time-accurate computations. Daso et al. ${ }^{16}$ did not report any three-dimensionality effects for the zero angle of attack cases, and hence assumption of an axisymmetric flow is valid. With triangular meshes being the preferred grid topology for the CESE framework, unstructured triangular meshes, generated by GRIDGEN ${ }^{\circledR}$ and University of Alabama at Birmingham's in-house code, the Mixed-Element Grid Generator in 3 Dimensions (MEGG3D), ${ }^{28,}{ }^{29}$ have been utilized in this study. The meshes for all the computations here have been generated based on our previous experience. ${ }^{23,30}$ As a result, no grid convergence study has been demonstrated as part of this work. The original mesh employed for the baseline (no jet) computations contained approximately 280,000 triangular cells and 141,000 nodes. The meshes employed for all of the other computations (those with the nozzles) contained approximately 430,000 triangular cells and 216,000 nodes. All of the meshes employed are clustered near the CEV surface and nozzle wall to maintain sufficient grid resolution for predicting heat transfer. Overall schematics of the computational setup (boundary conditions along with the locations of some key probes employed to monitor the flow) and the close-up views of the mesh employed are provided in Figs. 4(a) and (b) respectively.

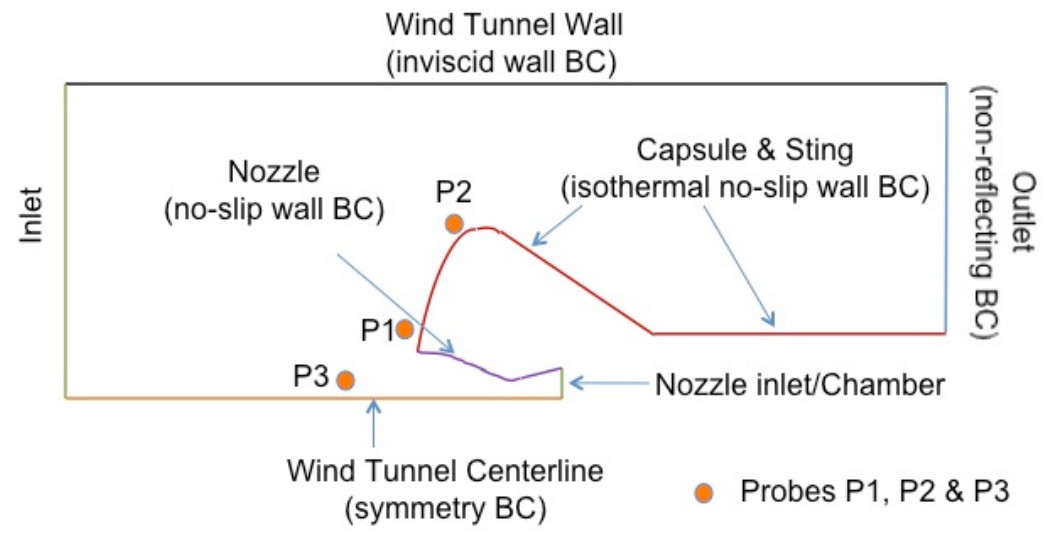

(a) Schematics and boundary conditions

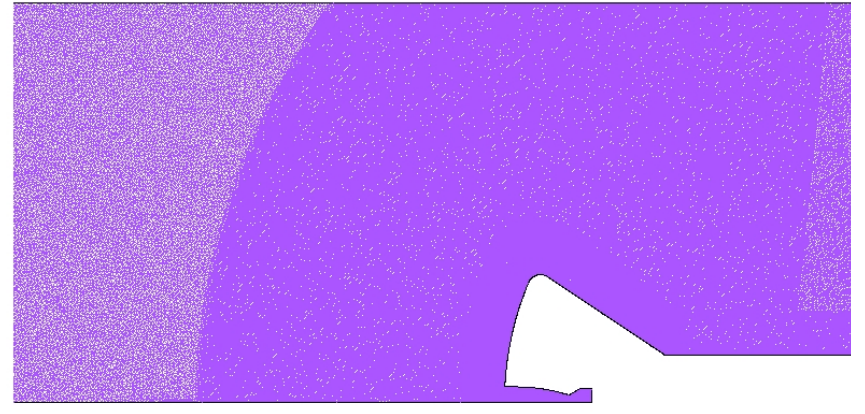

Mesh overview

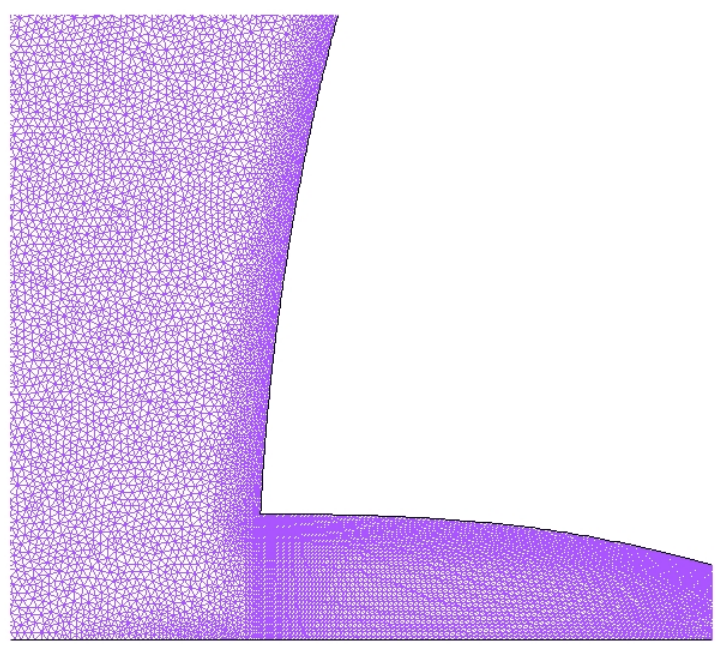

Mesh close-up

(b) Mesh details

Fig. 4. Computational set-up and mesh details.

\section{A. Baseline computations (No-jet case)}

Figre 5 shows the steady flowfield obtained for the baseline case without the counterflowing jet. The computed Mach number, temperature, and density gradient contours are shown along with a comparison against Schlieren images obtained 
from Daso et al. ${ }^{16}$ It can be seen that the computed shock standoff distance is in good agreement with the experiment. In addition to the bow shock, the separation region near the capsule aftbody-sting junction, the reflected shock off the wind tunnel wall, its interaction with the shock emanating from the separation region in the capsule aftbody, as well as the boundary layer above the sting are all clearly visible from the computational results. The time-history of the integrated heat flux on the capsule face, integrated from the line of symmetry to the capsule's shoulder, neglecting the aftbody and sting portion) is shown in Fig. 6 and is indicative of the steady-state nature of the flow. Negative heat-flux indicates cooling of the surface and this behavior is expected as the capsule wall has been set to $297 \mathrm{~K}$.

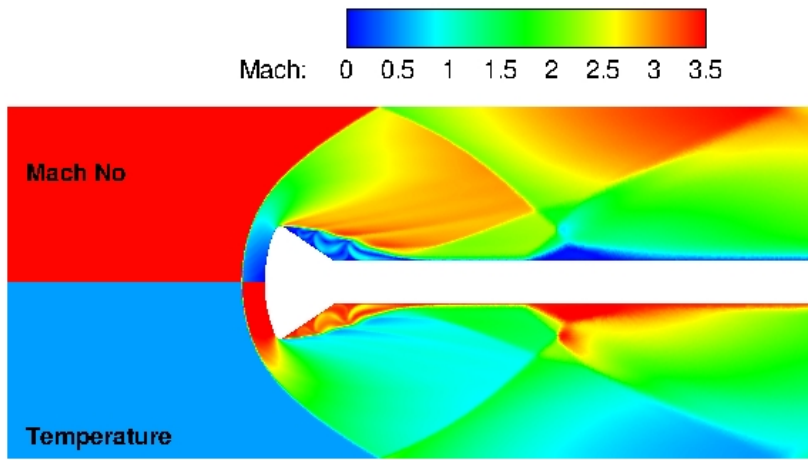

Temperature $(\mathrm{K})$ :

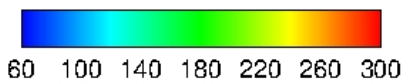

(a) Mach number and temperature contour plot

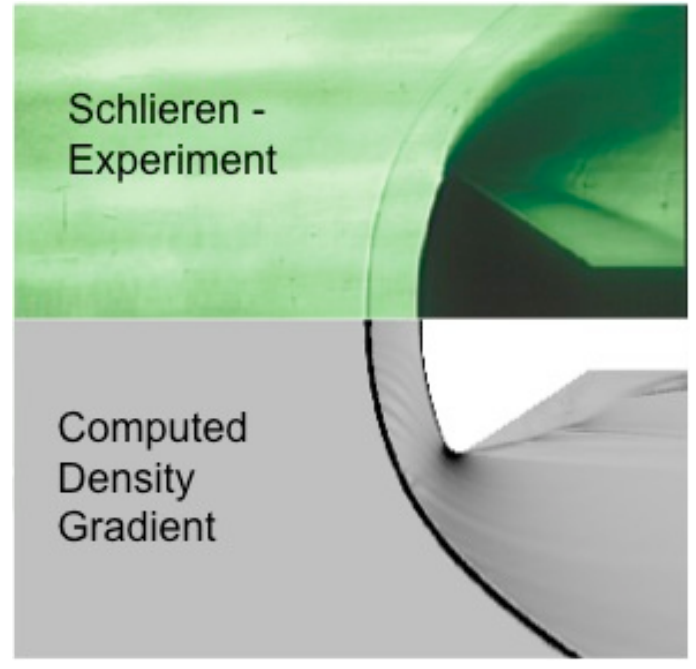

(b) Experimental Schlieren versus computed density gradient

Fig. 5. Computed Mach, temperature and density gradient contours for baseline geometry with no jet $\left(M_{\infty}=3.48\right)$.

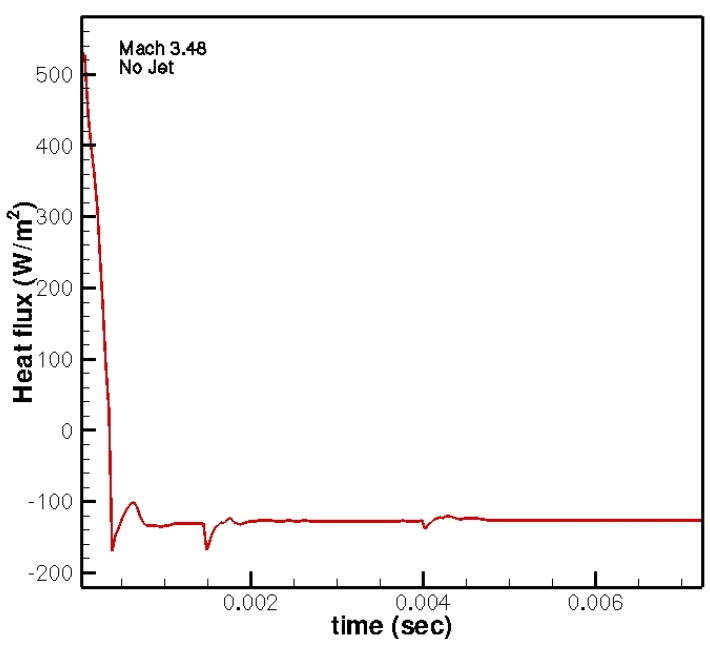

Fig. 6. Time history of the heat flux integrated on the capsule face from the centerline to $\left(M_{\infty}=3.48\right.$; no jet).

\section{B. Mach 2.98 Jet Nozzle}

Mach 2.98 jet nozzle results are presented ahead of the Mach 2.44 jet nozzle, as Schlieren images from Daso et al. ${ }^{16}$ are available for comparison. Also note that, Schlieren images from Daso et al. ${ }^{16}$ are available for the N2mf1, M2mf2 and $\mathrm{N} 2 \mathrm{mf} 5$ runs alone and not for $\mathrm{N} 2 \mathrm{mf} 3$ and $\mathrm{N} 2 \mathrm{mf} 4$, as these two test conditions were not part of the experiments done by Daso et al. ${ }^{16}$ The two test conditions, $\mathrm{N} 2 \mathrm{mf} 3$ and $\mathrm{N} 2 \mathrm{mf} 4$ respectively, were constructed by the authors with the aim of obtaining a clearer understanding of the transition from LPM to SPM, as Daso et al. ${ }^{16}$ had pointed out in their study the quick transition of the jet from the LPM configuration to the SPM configuration for flow rates beyond that corresponding to the $\mathrm{N} 2 \mathrm{mf} 2$ case $(0.0453 \mathrm{~kg} / \mathrm{s})$. For this reason, test conditions $\mathrm{N} 2 \mathrm{mf} 3$ and $\mathrm{N} 2 \mathrm{mf} 4$ are designed to have intermediate flow rates that are slightly larger than that of the $\mathrm{N} 2 \mathrm{mf} 2$ run but smaller than the $\mathrm{N} 2 \mathrm{mf} 5$ run. 
As indicated in Figs. 7(a)-(c), with increase in mass flow rate, the jet's interaction with the bow shock increases. As a consequence, the bow shock gets pushed further away from the capsule surface, resulting in a longer shock standoff distance than that corresponding to the test conditions with no jet. The level of jet penetration, however, varies quite drastically with the mass flow rate. For smaller flow rates, the jet begins to penetrate the bow shock; as the mass flow rate increases, this penetration increases substantially, that it begins to completely disperse the strength of the shock while simultaneously pushing the shock further away from the capsule surface, resulting in the unstable LPM configuration. Finally, as the jet mass flow rate increases beyond a threshold level, the jet-bow shock interaction suddenly collapses back into the stable SPM condition, wherein the jet does not penetrate the bow shock, but rather expands to form an effective capsule with a less blunt surface to the incoming supersonic freestream. As seen from Figs. 7(a) and (c), the CESE computations are able to capture all the features of the jet interaction observed in the Schlieren images, including the correct shock standoff distance. The dispersed shock observed in $\mathrm{N} 2 \mathrm{mf} 2$ computations is not shown well in the experimental Schlieren images; and Daso et al. ${ }^{16}$ mentioned this aspect in their work. In an additional study ${ }^{16}$ done through employment of a high-speed camera, they were able to observe more details of this interaction mode and the computed results are in line with the observed trend. As noted by Daso et al., ${ }^{16}$ cases $\mathrm{N} 2 \mathrm{mf} 1$ and $\mathrm{N} 2 \mathrm{mf} 2$, corresponding to LPM jet interaction, were highly unsteady. Cases N2mf3 and N2mf4 constructed in this study were also found to be unsteady. In contrast, the N2mf5 case was largely stable with only mild unsteadiness. This indicates that there might exist a critical jet mass flow rate for the LPM. Time series from the various probes placed in the computations provide more details on this aspect and will be discussed later in this paper.

The LPM jet interactions are highly unstable. For the N2mf2 case, the flow structure is characterized by the movement of the bow shock location with time between $\mathrm{x}=0.25 \mathrm{~m}$ to $\mathrm{x}=0.076 \mathrm{~m}$ (see Fig.8) at the centerline without settling in one axial location. The bow shock seemed to settle into a quasi-steady state at several locations only to collapse back (resembling an SPM like configuration) or get pushed upstream from that location. This behavior as well as the subsequent transition from LPM to SPM at a higher jet mass flow rate is a direct outcome of the jet structures shown in Figs. 7(a) -(c). At the smaller flow rates (Figs. 7(a) and 7(b)), the jet structure is more like that shown in Fig. 1(b), while that for SPM (Fig. $7(\mathrm{c})$ ) resembles Fig. 1(a). The differences in these flow structures can be attributed to the difference in the ratio of the static pressure of the jet at the nozzle exit plane and the ambient static pressure outside the nozzle exit $\left(P_{j} / P_{\mathrm{amb}}\right)$. A jet with $P_{j} / P_{\mathrm{amb}}>1$ is characterized as an under-expanded jet, while for $P_{j} / P_{\mathrm{amb}}>1$, the jet is said to be highly underexpanded. In a slightly under-expanded jet, as the flow expands into the ambient gas with low-pressures, expansion fans are created at the nozzle exit. The free jet boundary reflects these expansion fans as compression waves, which then coalesce to form an incident shock. The incident shock (see Fig. 1(b)) then undergoes a regular reflection (without forming a Mach disk) at the jet axis. The resulting diverging shock, once again, interacts with the jet boundary creating another incident shock and the process repeats resulting in the familiar diamond shock-cell structure. The consecutive deceleration by the shock cell slows down the jet plume until it stagnates at the free stagnation point. At this point, the jet plume meets the post normal shock flow near the centerline, with both their total pressures reaching a value of $P_{0 f}$. It is understood that a normal shock is not stable if it occurs in the decelerating region of a flow. ${ }^{11}$ The LPM jets belong to this class; the under-expanded jet adjusts itself to get the terminating normal shock to end up in the accelerating flow region. In the case of the highly under-expanded jet, the incident shock rather than undergoing a regular reflection at the jet axis, instead forms a Mach disk. The Mach disk is quickly able to decelerate the supersonic jet to come into equilibrium with the ambient flowfield. Moreover, since this normal shock remains within the accelerating region of the jet, the flow remains very stable. Larger jet mass flow rates are often a consequence of larger jet-to-freestream total pressure ratios $\left(P_{0 j} / P_{0}\right)$, and as a result, the SPM jet interactions found in these cases comply well with this highly under-expanded jet behavior. Fig. 8, along with Figs. 9(a)(d), where the results for several jet mass flow rates are shown, clearly demonstrates the aforementioned phenomenon. The corresponding Mach number and static pressure distributions along the jet axis for various jet flow rates are shown in Figs. 10(a)-10(b). The counterflowing jet and the freestream meet at the stagnation point after each undergoes a strong normal shock for $\mathrm{N} 2 \mathrm{mf} 4$ and $\mathrm{N} 2 \mathrm{mf} 5$. In contrast, the counterflowing jet undergoes additional shocks before they meet for $\mathrm{N} 2 \mathrm{mf} 2$ and $\mathrm{N} 2 \mathrm{mf} 3$. In the extreme case of $\mathrm{N} 2 \mathrm{mf}$, three strong shocks are clearly visible.

Several probes were placed in the computational domain to monitor the flow and record time history data. Among those, we show results for probes P1, P2 and P3 that are shown in Fig. 4(a). Probe P1 was placed in the region just above the nozzle exit (in the recirculation region; $\mathrm{x}=-0.0178 \mathrm{~m}$ and $\mathrm{y}=0.0367 \mathrm{~m}$ ), while Probe $\mathrm{P} 2$ was closer to the shoulder of the capsule face $(\mathrm{x}=0.0 \mathrm{~m}$ and $\mathrm{y}=0.042 \mathrm{~m})$. Probe P3 was at an axial location outside the nozzle and within the jet's core ( $\mathrm{x}$ $=-0.001 \mathrm{~m}$ and $\mathrm{y}=0.0002 \mathrm{~m}$ ). The nozzle exit along the line of symmetry was at location $\mathrm{x}=0.0$ and $\mathrm{y}=0.0$. Note that, results from Probe P3 will only be shown for LPM jets, as it is expected to be indicative of the dynamic changes occurring to the jet behavior. In Fig. 11, the time history of the static pressure and its spectra are shown for the LPM jet (N2mf2) and the SPM jet (N2mf5). As can be observed from Fig. 11(a), the time history data from all the three probes indicate a highly unsteady flow phenomenon that is expected of the LPM. The frequency spectra feature a broad-band structure without any distinct dominating modes. Multiple peaks seen here could be indicative of the quasi-steadiness achieved in the transient. 

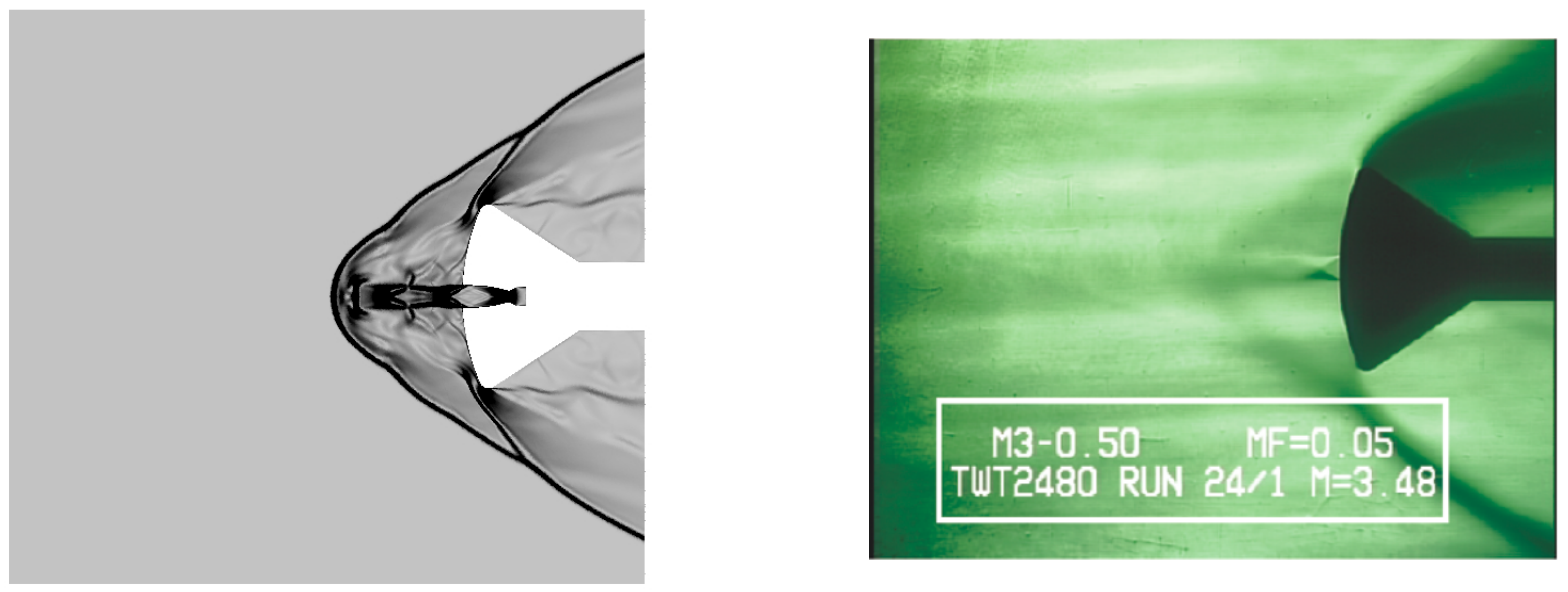

(a) N2mf1 $\left(\dot{m}_{j}=0.0227 \mathrm{~kg} / \mathrm{s}\right)$
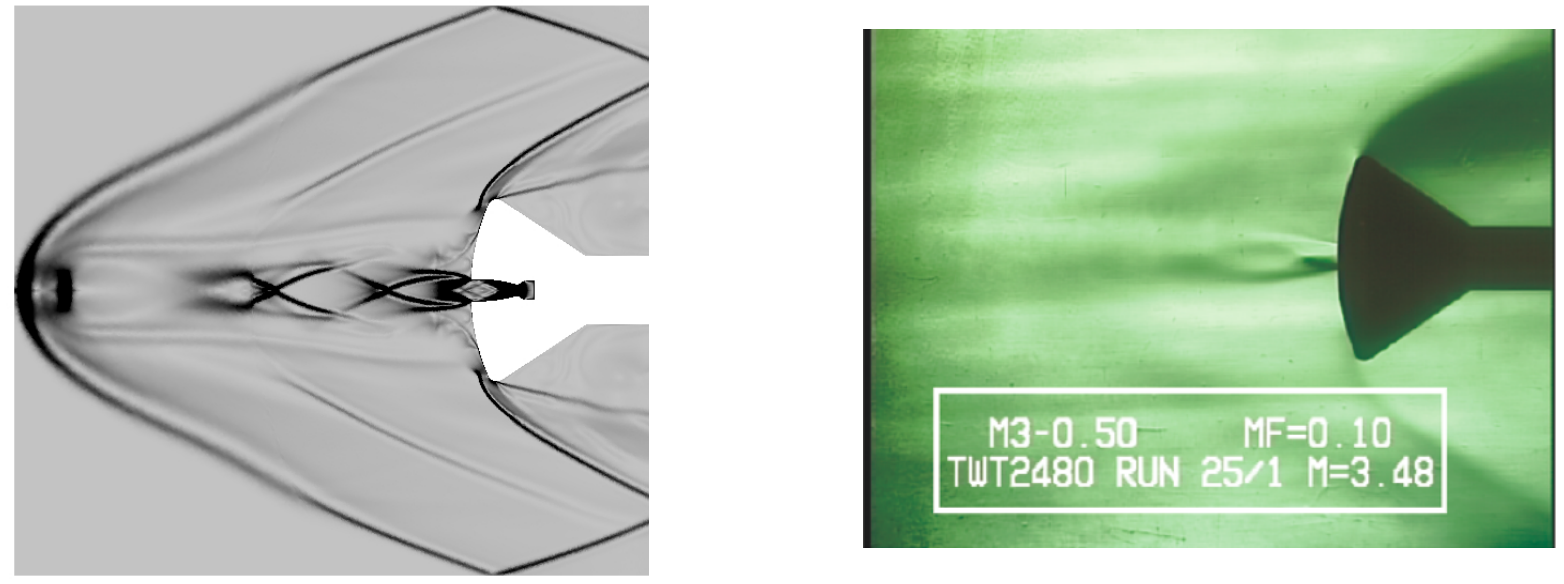

(b) $\mathrm{N} 2 \mathrm{mf} 2\left(\dot{m}_{j}=0.0453 \mathrm{~kg} / \mathrm{s}\right)$
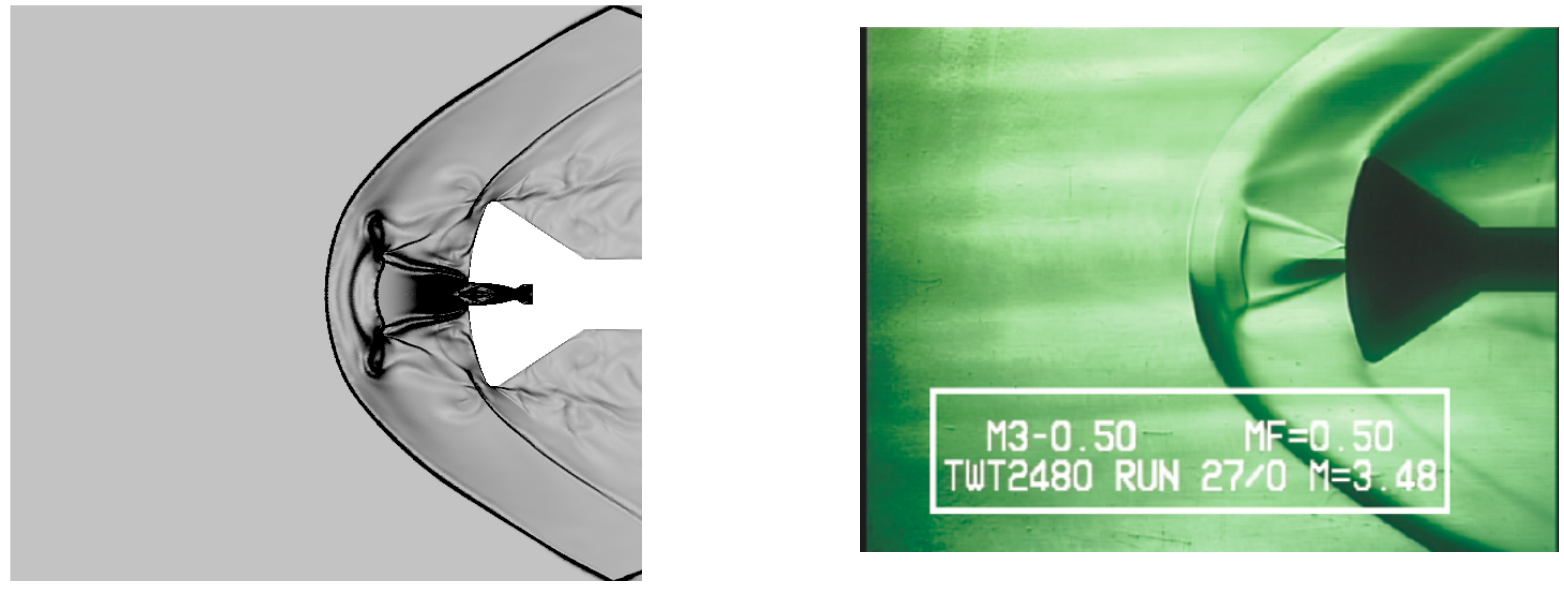

(c) $\mathrm{N} 2 \mathrm{mf} 5\left(\dot{m}_{j}=0.2268 \mathrm{~kg} / \mathrm{s}\right)$

Fig. 7. Effects of flow rate on the interaction of the counterflowing jet with freestream and $M_{j}=\mathbf{2 . 9 8}$; computed density gradient versus experimental Schlieren image. 
$\mathrm{t}=0.0004 \mathrm{~s}$

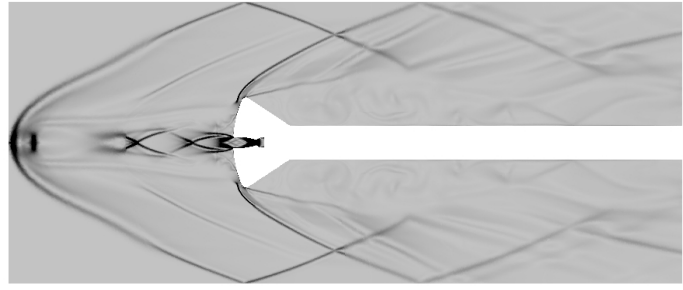

$t=0.003 s$

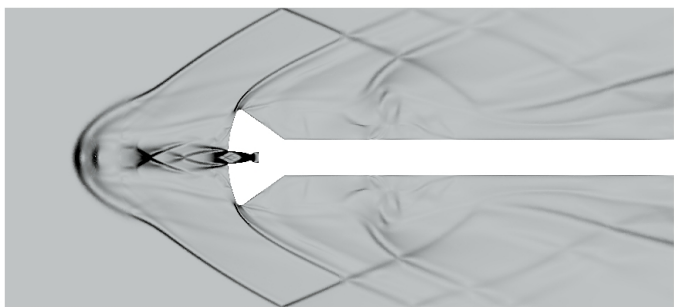

$\mathrm{t}=0.006 \mathrm{~s}$

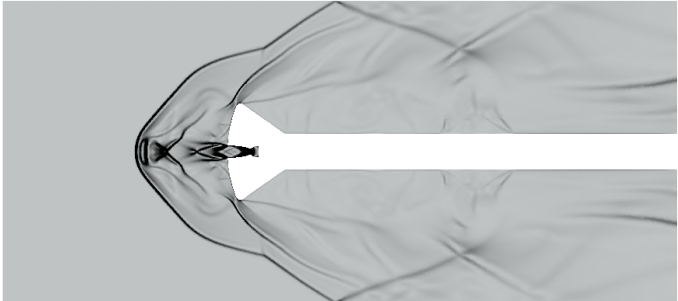

$\mathrm{t}=0.008 \mathrm{~s}$

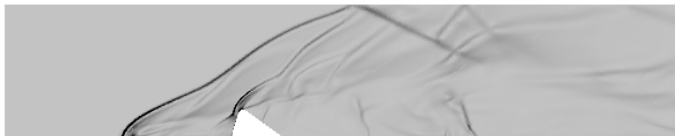

(3) $35 \times$
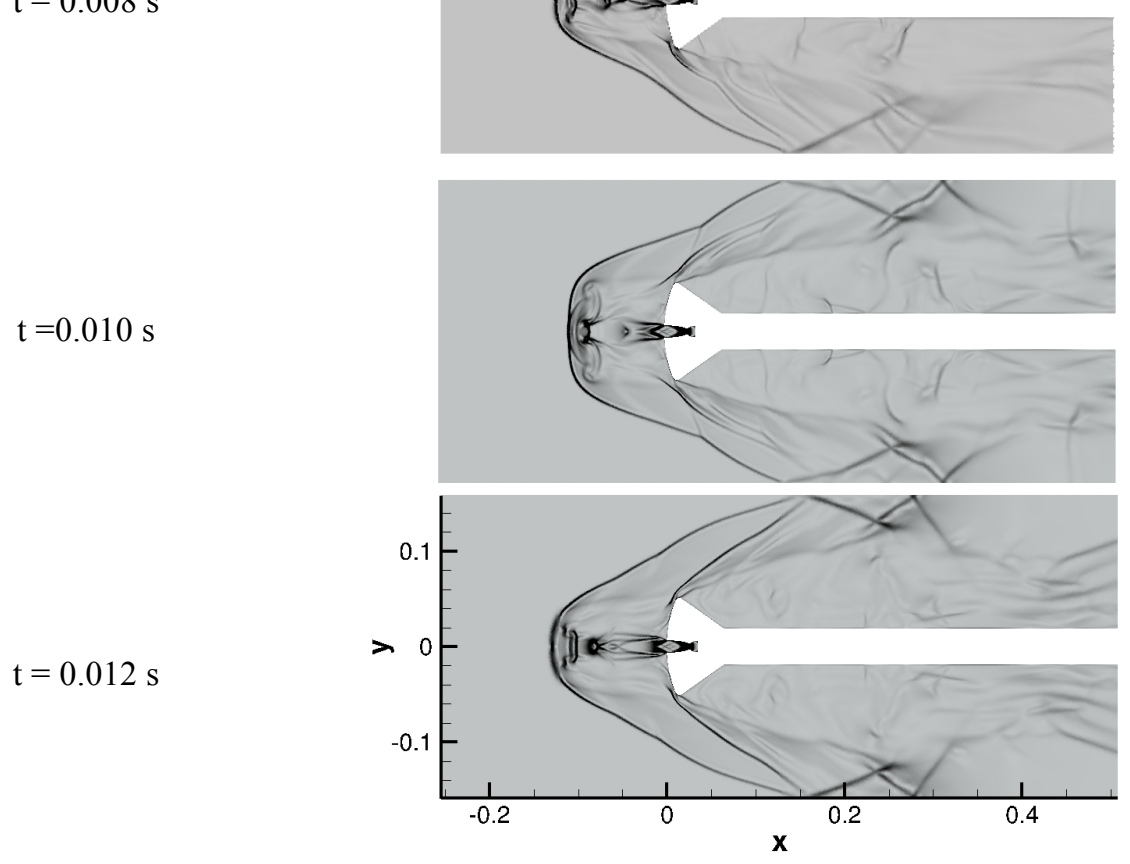

Fig. 8. Time sequence of the computed density gradient contour images for the LPM jet (N2mf2). 


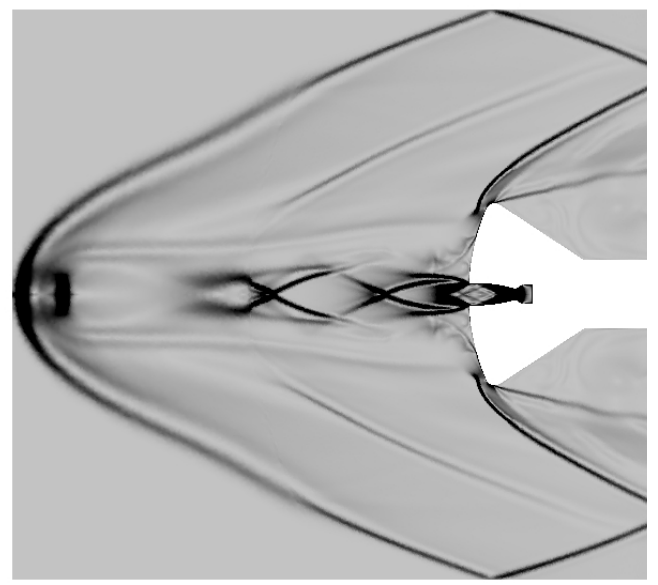

(a) $\mathrm{N} 2 \mathrm{mf} 2\left(\dot{m}_{j}=0.0453 \mathrm{~kg} / \mathrm{s}\right)$

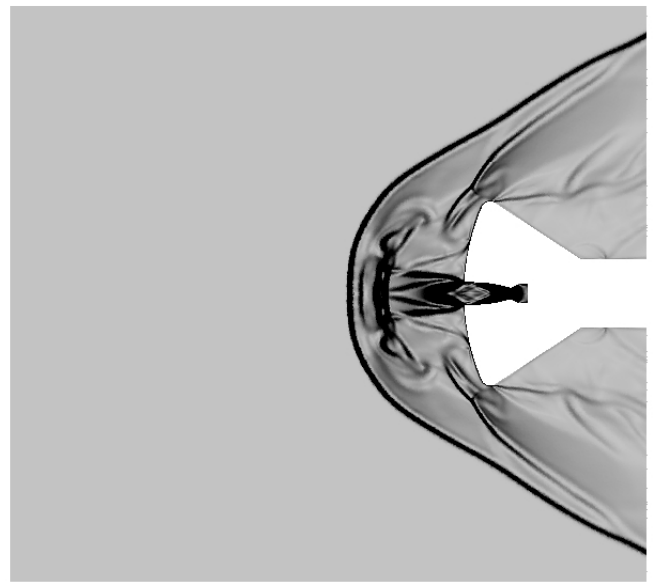

(c) $\mathrm{N} 2 \mathrm{mf} 4\left(\dot{m}_{j}=0.0907 \mathrm{~kg} / \mathrm{s}\right)$

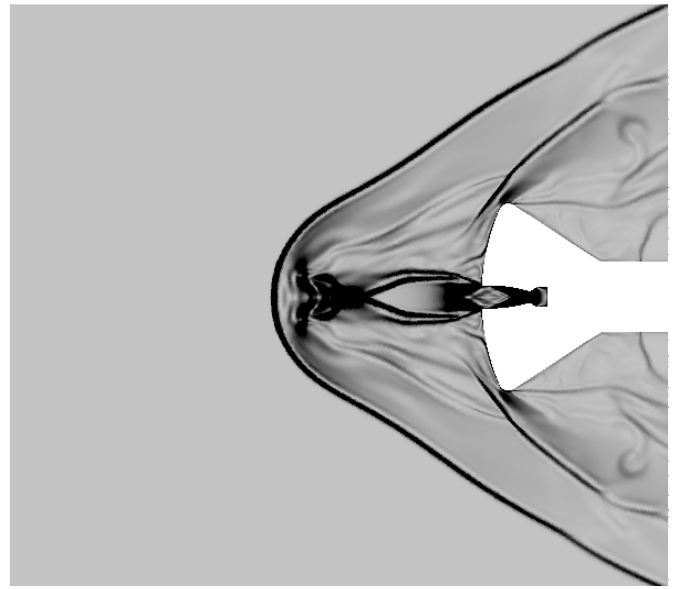

(b) $\mathrm{N} 2 \mathrm{mf} 3\left(\dot{m}_{j}=0.0680 \mathrm{~kg} / \mathrm{s}\right)$

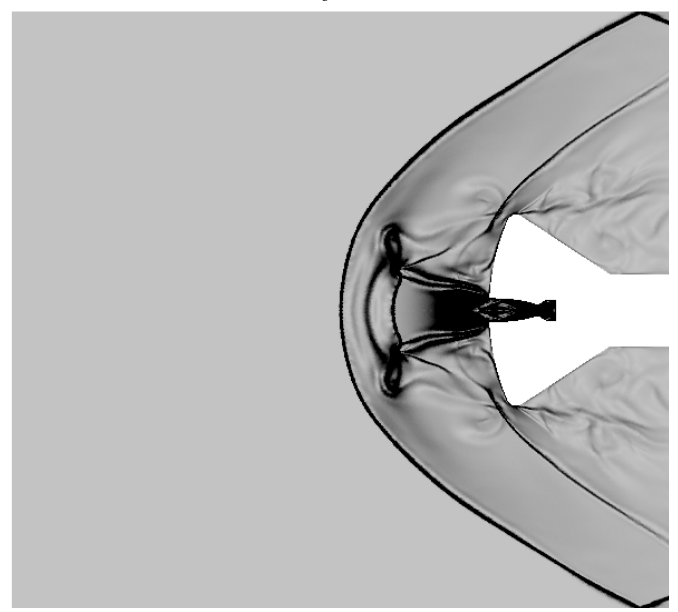

(d) $\mathrm{N} 2 \mathrm{mf} 5\left(\dot{m}_{j}=0.2268 \mathrm{~kg} / \mathrm{s}\right)$

Fig. 9. Influence of jet flow rate in the transition from LPM to SPM (Density gradient contours).
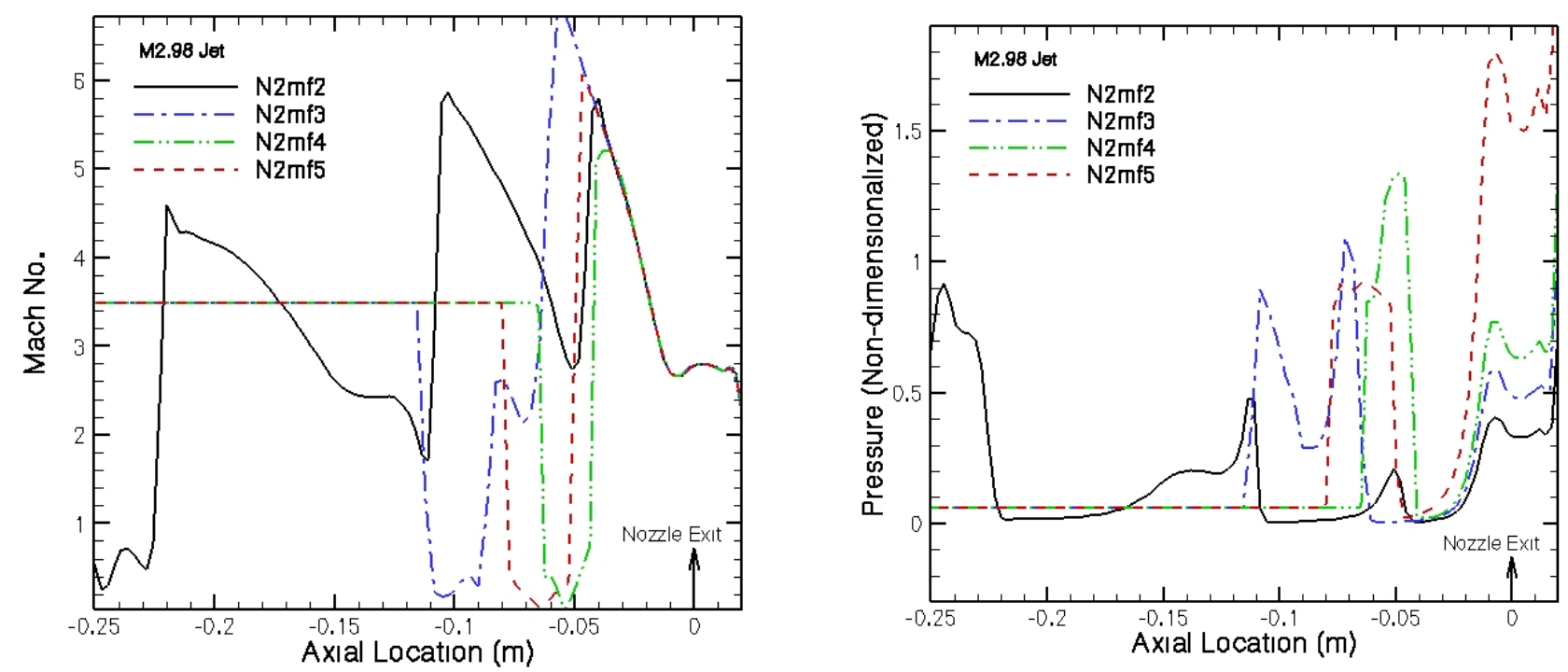

Fig. 10. Variation of Mach number and pressure across the jet centerline/axis for various jet flow rates. 

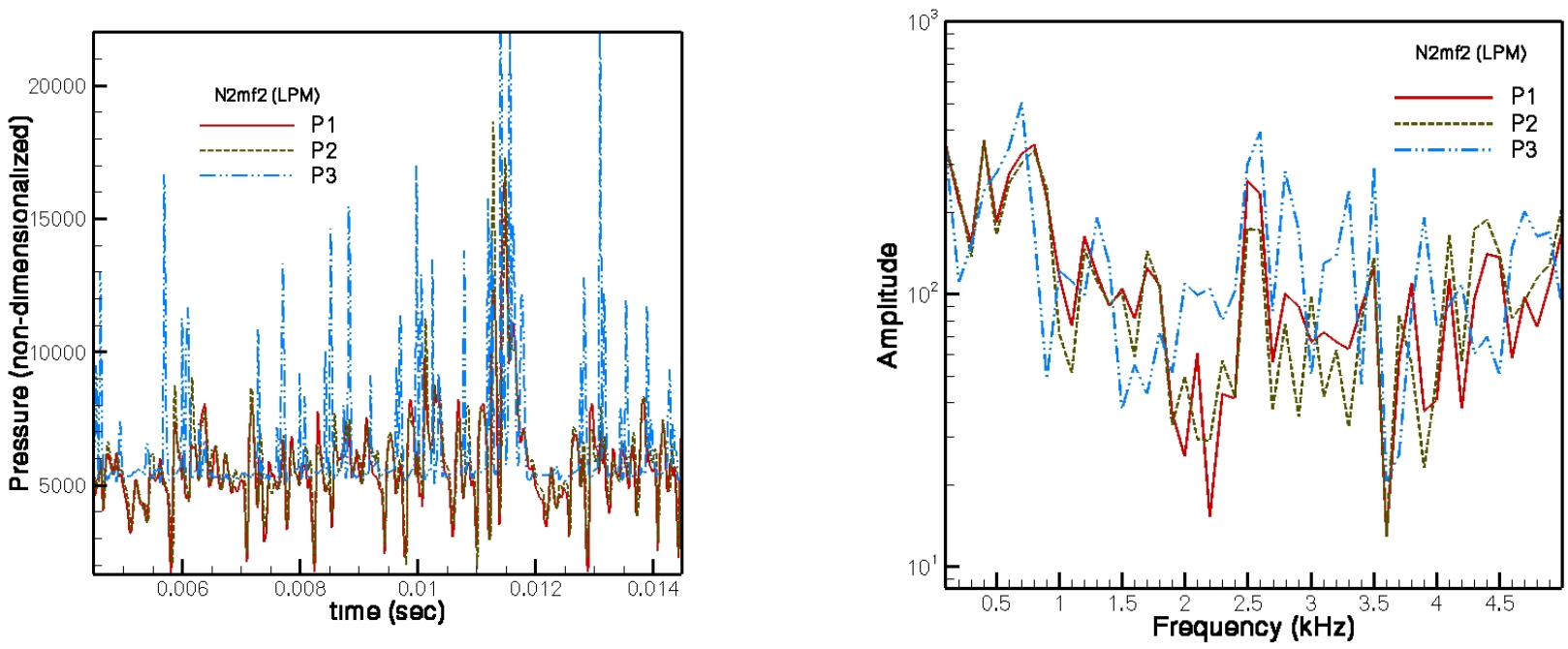

(a) N2mf2 (LPM)
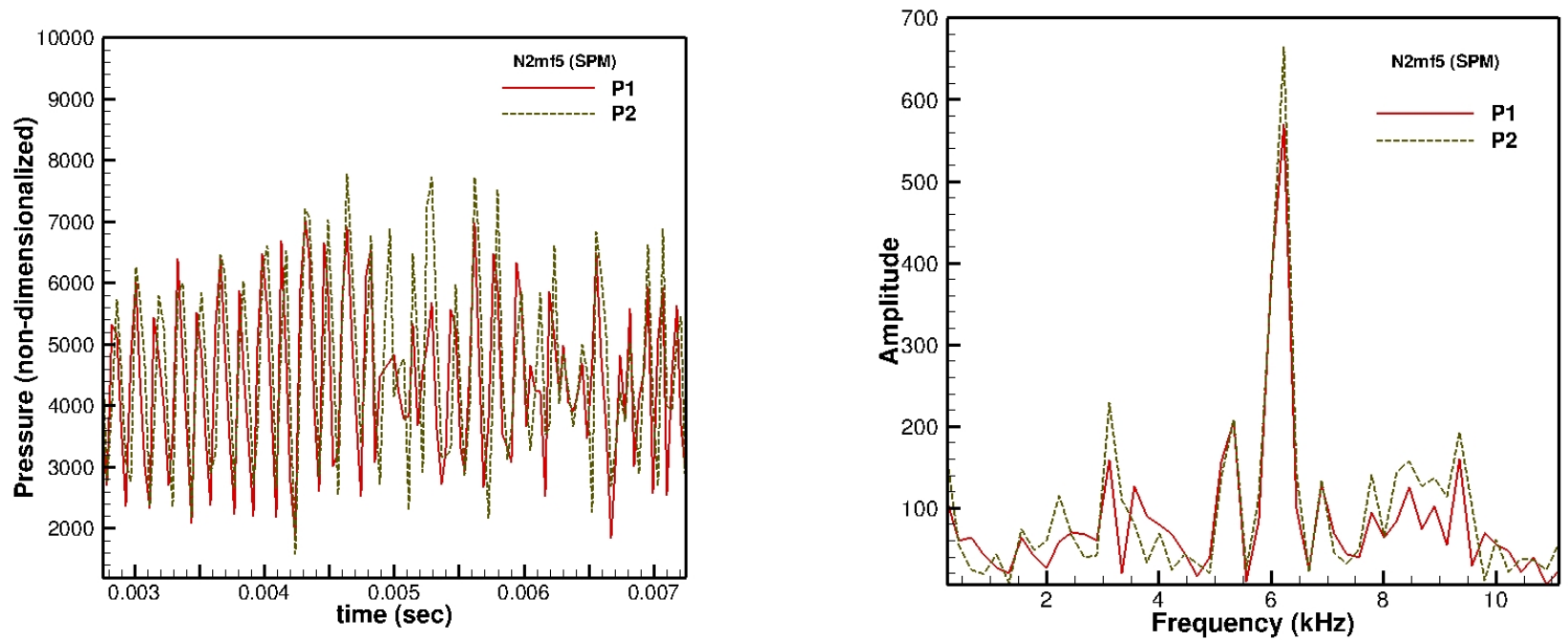

(b) N2mf5 (SPM)

Fig. 11. Time history and spectra of the pressure at various probes placed in the computational domain.

The overall broad-band structure of the spectra only confirms the complexity of the process involved in LPM jets, indicating the instability contributions from the oscillating jet, jet shear layer and the bow shock. Data correlation after a longer time integration is expected to provide more information on the flow. In contrast, for the SPM jet (see Fig. 11 (b)), probes P1 and P2 only experience a mild fluctuation and have a distinct dominating mode. Based on theory and the computational results, these fluctuations are a result of a small jet stream emanating in the region where the jet shear layer meets the Mach disk. This jet stream oscillates periodically, affecting the curvature of the shock (shock standoff location is unaffected) and also periodically impinges onto the shoulder of the capsule. Probes P1 and P2 indicate a dominant frequency of around $6 \mathrm{kHz}$.

\section{A. Mach 2.44 Jet Nozzle}

Similar to the Mach 2.98 jet cases, cases N1mf1, N1mf2 and N1mf5 (see Table 1) alone were based on actual test conditions from Ref. 16. For reasons explained earlier, additional cases, N1mf3 and N1mf4 respectively, were designed to give slightly higher flow rates than test case N1mf2. Based on the computational results shown in Figs. 12 (a)-(e), the condition for maximum jet penetration occurs (N1mf1) at lower mass flow rates than the Mach 2.98 jet case. This indicates that for lower jet speeds, LPM occurs at much lower jet-to-freestream total pressure ratios, compared to higher speed jets. The jets exhibit similar features as those for the Mach 2.98 nozzle. Similar to the previous case at a higher jet mass flow rate, the LPM jets had substantial unsteadiness resulting in oscillation of the bow shock position as the jets reduce total pressure to match that of the free stagnation point through a series of shock compressions and expansions (see pressure and 


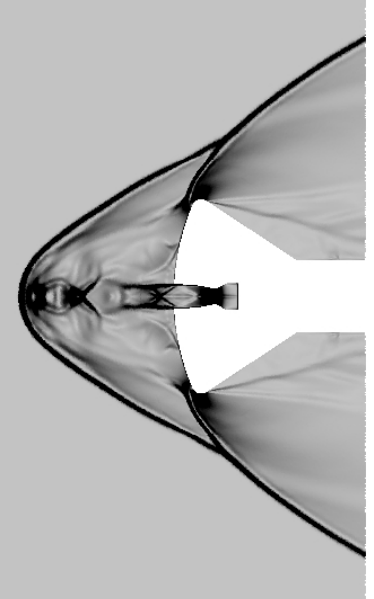

(a) N1mf1 $\left(\dot{m}_{j}=0.0227 \mathrm{~kg} / \mathrm{s}\right)$

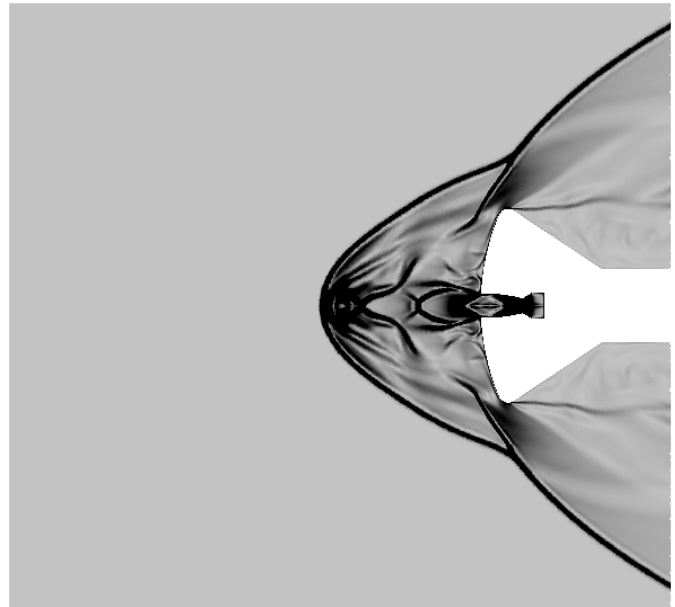

(c) N1mf3 $\left(\dot{m}_{j}=0.0680 \mathrm{~kg} / \mathrm{s}\right)$

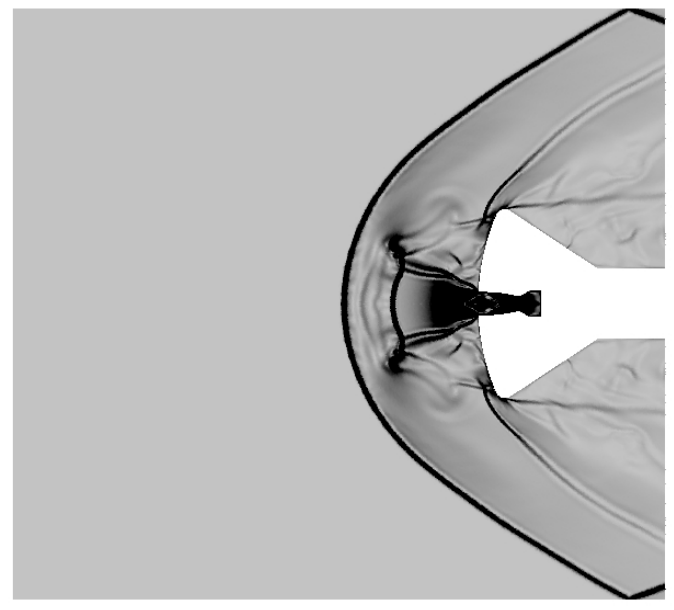

(e) $\mathrm{N} 1 \mathrm{mf} 5\left(\dot{m}_{j}=0.2268 \mathrm{~kg} / \mathrm{s}\right)$

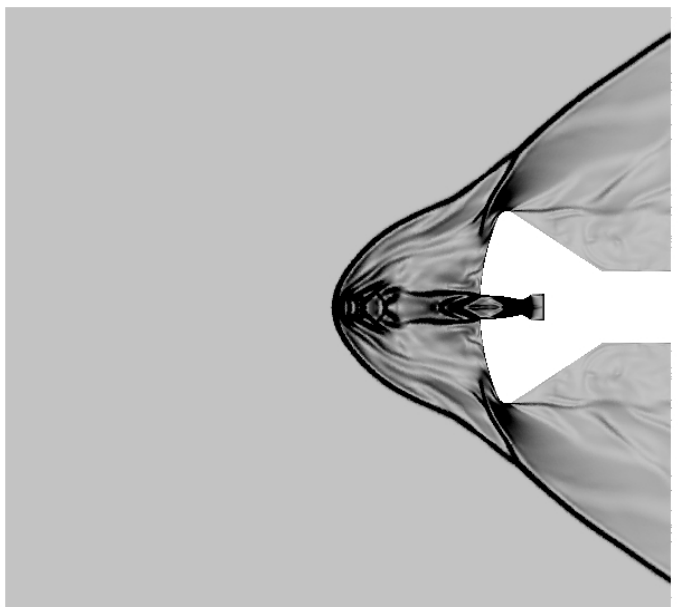

(b) N1mf2 $\left(\dot{m}_{j}=0.0453 \mathrm{~kg} / \mathrm{s}\right)$

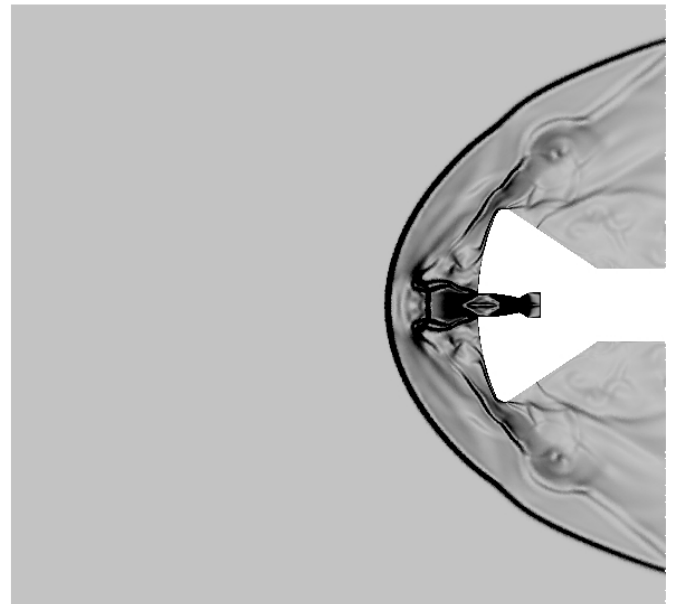

(d) N1mf4 $\left(\dot{m}_{j}=0.0907 \mathrm{~kg} / \mathrm{s}\right)$

Fig. 12. Effects of flow rate on the interaction of the counterflowing jet with freestream and $M_{j}=\mathbf{2 . 4 4}$ (computed density gradient). 

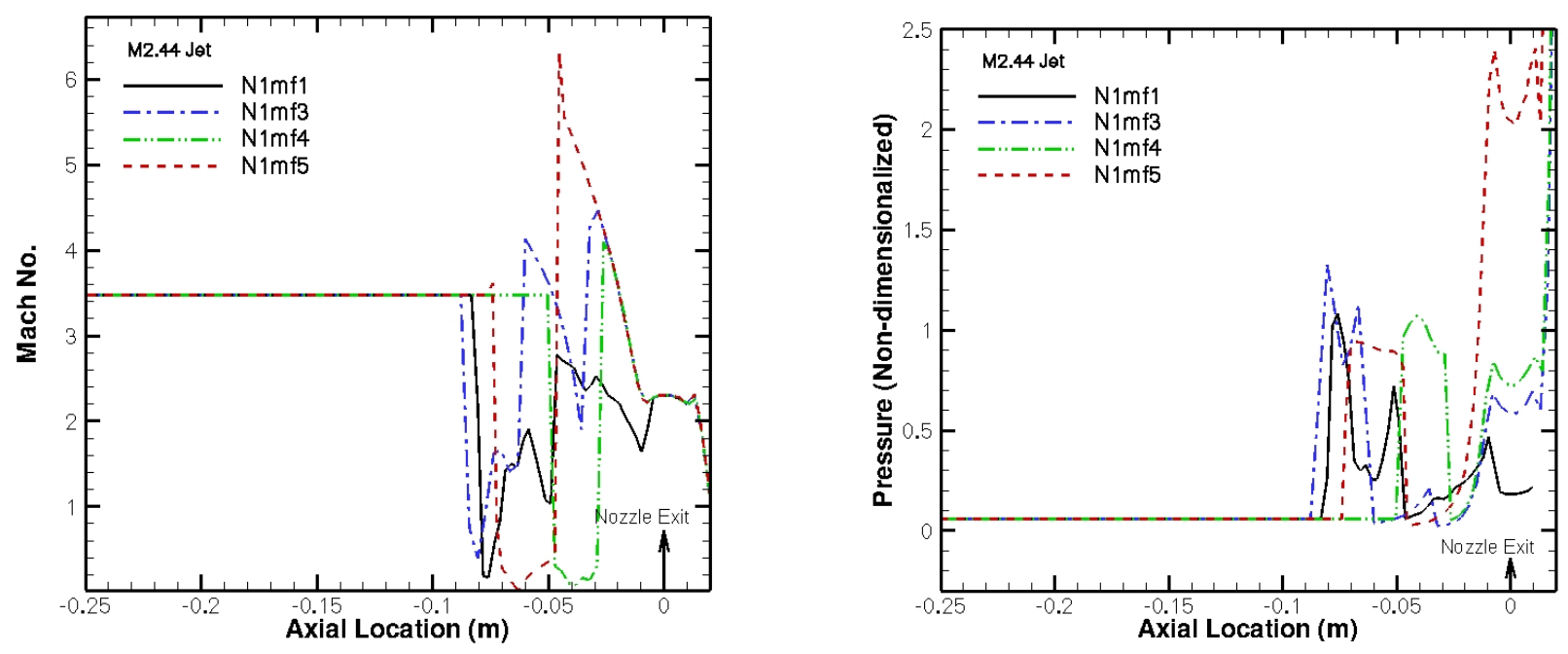

Fig. 13. Variation of Mach number and Pressure across the jet centerline/axis.
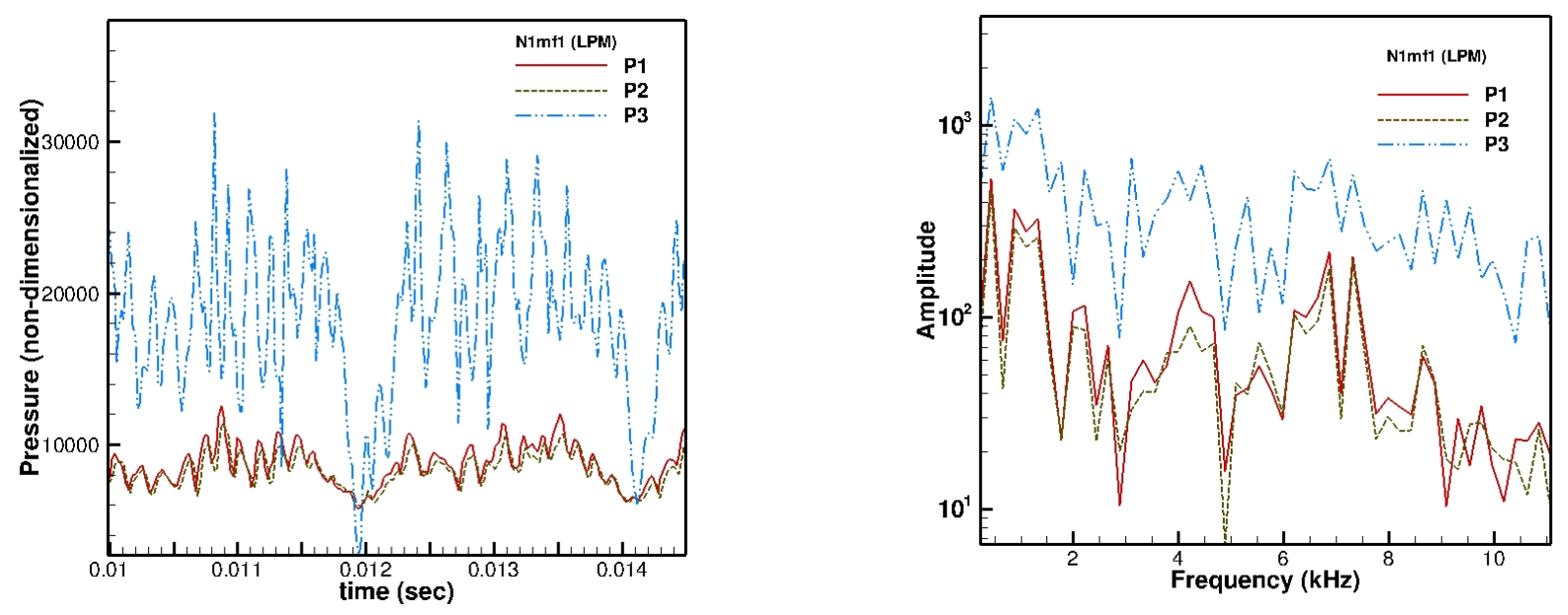

(a) N1mf1 (LPM)
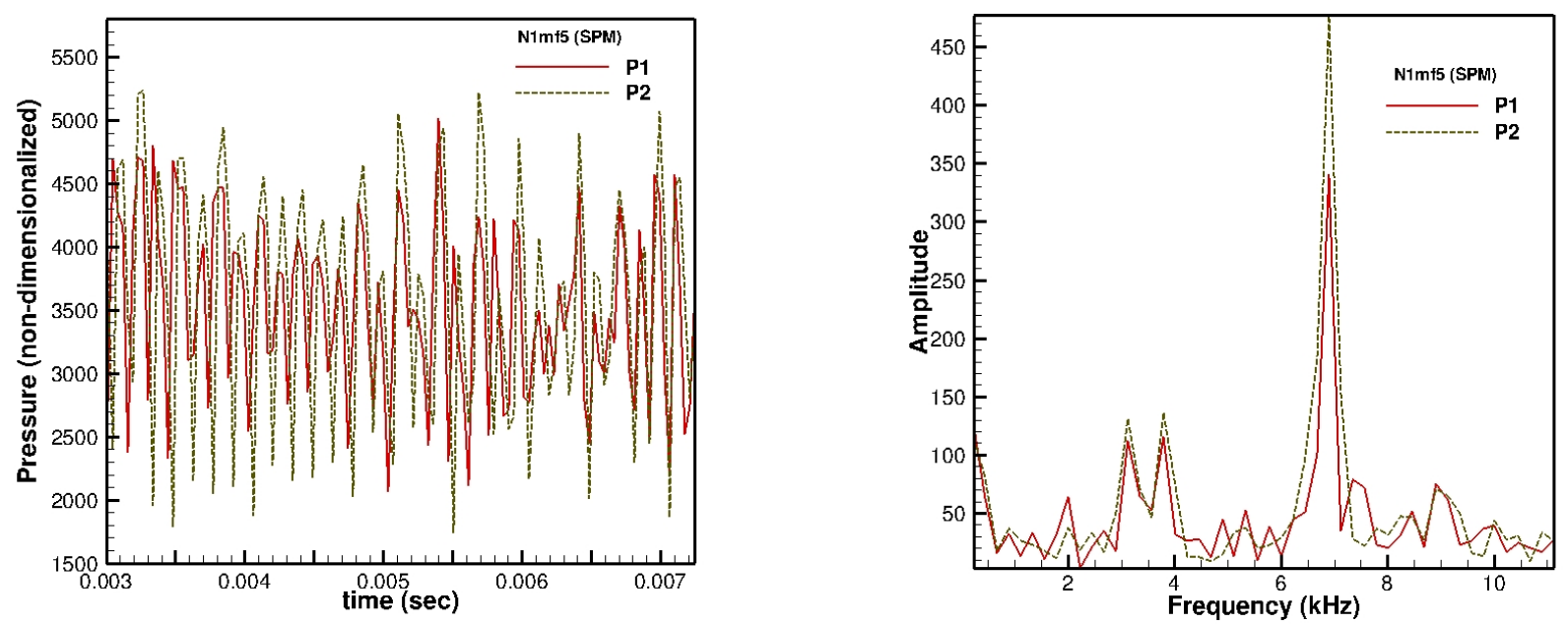

(b) N1mf5 (SPM)

Fig. 14. Time history and spectra of the pressure at various probes placed in the computational domain. 


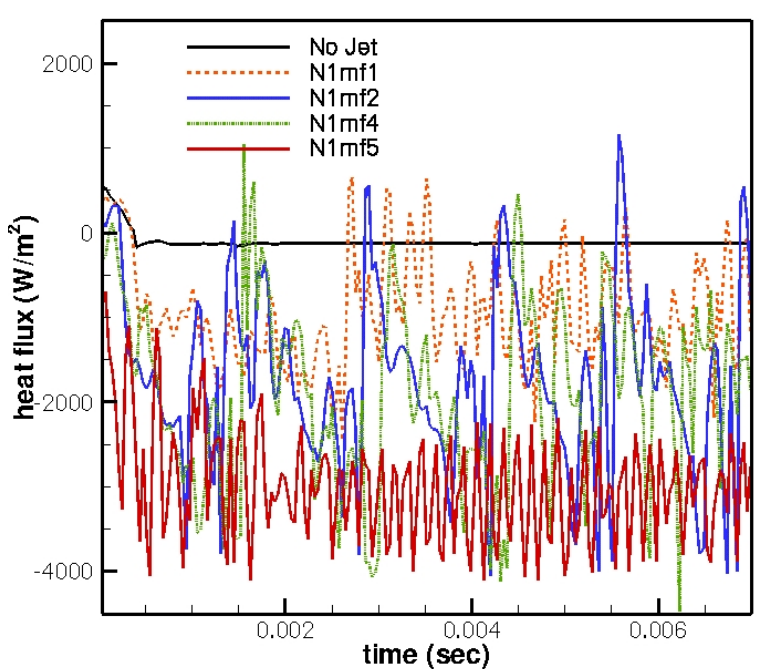

(a) Mach 2.44 Nozzle

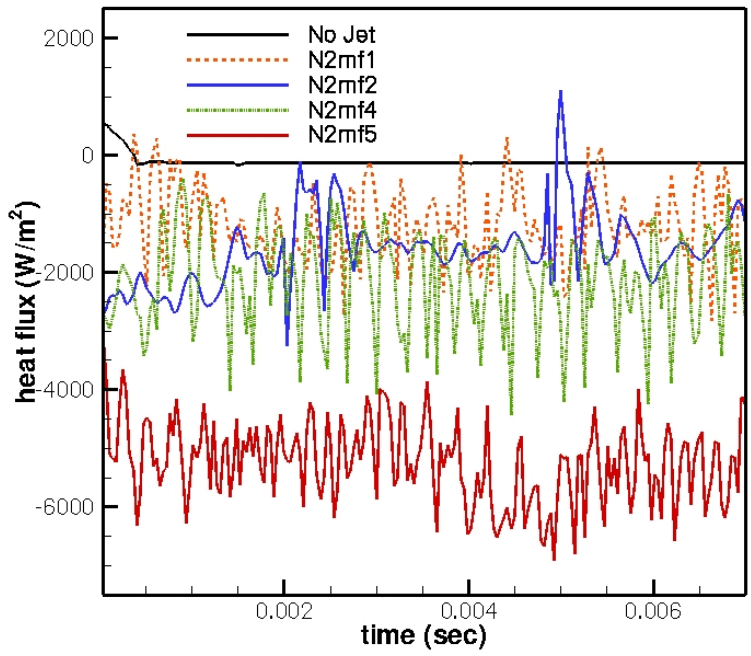

(b) Mach 2.98 Nozzle

Fig. 15. Time history of the integrated heat flux for the two nozzles (various jet mass flow rates).

The numerical results at Probes P1, P2 and P3 are shown in Fig. 14. It should be noted that these probes were positioned at the same locations as defined earlier and shown in Fig. 4(a). From Fig. 14(a) one can clearly see the unsteadiness in the LPM jet from the probe data and their spectra, which once again displays a very broad-band structure. The SPM jet spectra (Fig. 14(b)), on the other hand, exhibit only very small fluctuation levels because of its stable nature and possesses a clear and distinct mode around $7 \mathrm{kHz}$.

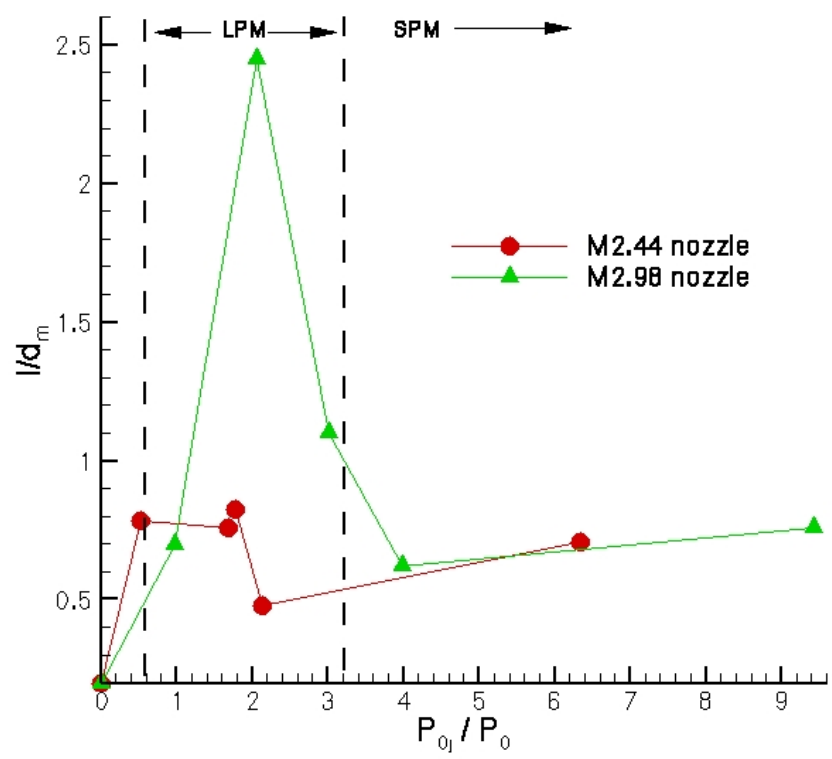

Fig. 16. Variation in shock displacement with jet speed and total pressure ratio.

Mach number distribution along the axis shown in Fig. 13). In addition, the SPM jet seemed to have a higher spreading angle than that for the higher speed jet.

Although not discussed in detail here, one of the main purposes for employing counterflowing jets is to reduce the thermal loads on the capsule or to increase the drag forces as necessary for entry and other applications. Fig. 15 confirms the evidence that counterflowing jets with larger mass flow rates can greatly mitigate thermal loads on the capsule face. As the flow rate increases for both nozzles, more cooling was achieved as indicated by a more negative value of the on integrated heat flux. It can also be observed from Fig. 15 that LPM jets tend to result in greater fluctuation (large changes in amplitude) in heat loads on the capsule surface in comparison to SPM jets. 
Figure 16 summarizes the key observations from this study, by presenting the maximum shock standoff distance (normalized by the capsule diameter) against the jet-to-freestream total pressure ratio $P_{0 j} / P_{0}$. The total pressure ratio is a better parameter to utilize than the jet mass flow rates; the reason being that, when $M_{\infty}$ is fixed, $M_{j}$ and $P_{0 j} / P_{0}$ can uniquely determine the $P_{j} / P_{\mathrm{amb}}$ ratio, which can provide insights on the kind of expansion the jet is likely to undergo as it exits the nozzle. It is evident from Fig. 16 that as $P_{0 j} / P_{0}$ increases, the shock standoff distance (with respect to the baseline case with no jet) increases to a peak value and then begins to drop drastically until it reaches a value that is slightly larger than that for the test conditions with no jet. Beyond this, it remains constant or increases by a small amount. Romeo and Sterrett ${ }^{31}$ have also previously reported this trend in their study. It was found that, as the jet speed increases, the $P_{0 j} / P_{0}$ ratio at which the maximum shock displacement takes place also increases. Based on these observations, it is possible to clearly delineate the LPM jet from the SPM jet. However, more studies (both experimental and computational) are needed to arrive at a specific criterion for the occurrence of LPM to SPM transition. Also from previous studies ${ }^{31}$ it was observed that even with smaller angles of attack $\left(<5^{\circ}\right)$ the long penetration mode vanishes completely. However, Daso et al ${ }^{16}$ observed that the LPM jet can be present even for angles of attack up to $10^{\circ}$. Further studies are needed in order to better understand this aspect.

\section{Solution-based Mesh Refinement Method}

Given the complex nature of jet-shock interaction, the use of solution-based mesh refinement can improve the accuracy of numerical results and the computational efficiency. Preliminary computations of a grid refinement study have been performed for the axisymmetric configurations shown previously. The method used to construct solution-adopted meshes involves simple isotropic refinement to refine triangular and tetrahedral meshes, based on either the shock sensor proposed by Lovely and Haimes ${ }^{32}$ or the weight function by Soni et al. ${ }^{33}$ Basically, each triangular or tetrahedral element in the domain of interest is subdivided into four or eight elements, respectively. Note that the current approach does not have mesh coarsening abilities.
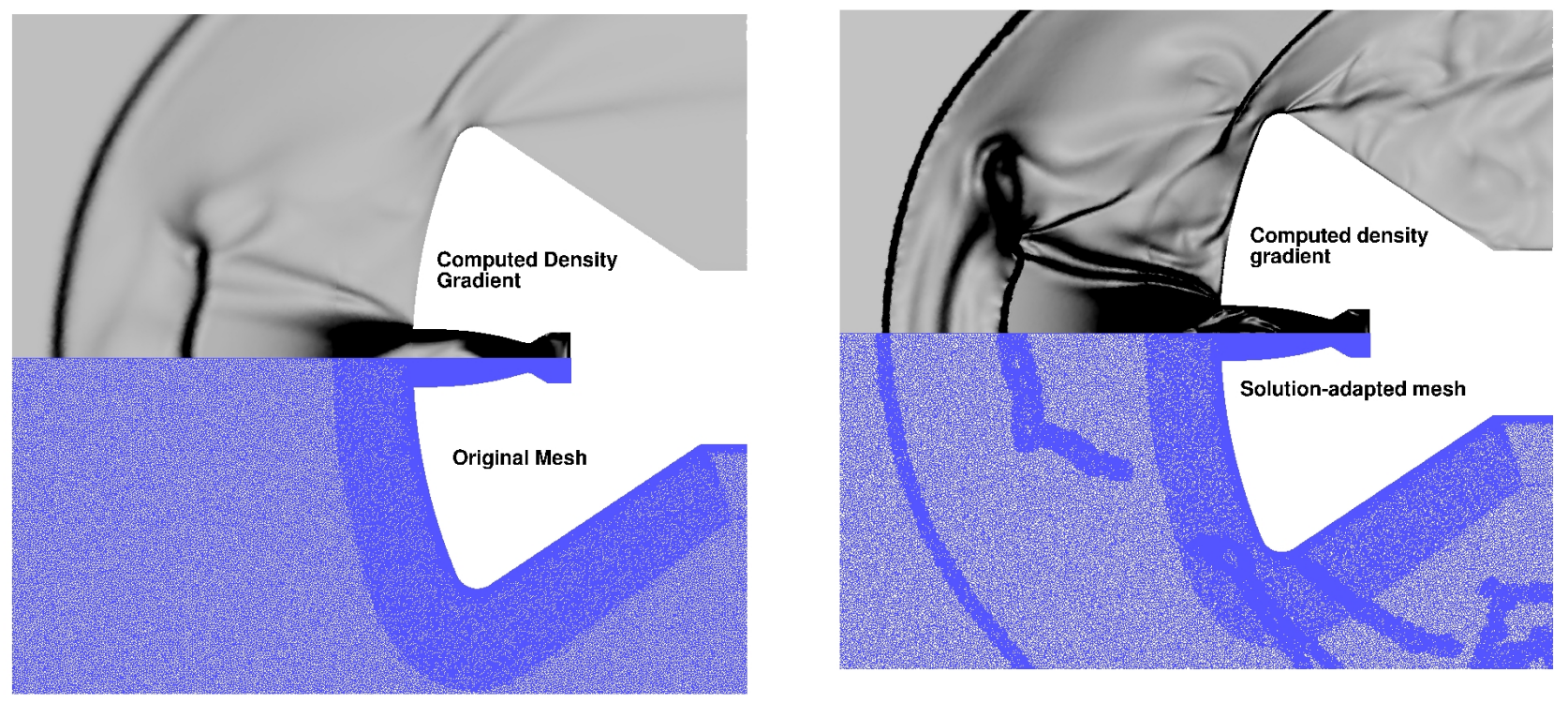

Fig. 17. Preliminary results of the solution-based mesh refinement method applied to the SPM jet.

Preliminary results from the solution-based refined mesh for the SPM jet interaction are shown in Fig. 17. As can be observed by comparing the original and modified meshes, a significant enhancement in grid resolution near the bow-shock as well as shock-boundary layer interaction regions was achieved by utilizing the solution-based mesh refinement approach. As a result the overall quality of the numerical result also improved. The overall grid count increases from 430,000 to 530,00 cells. The application of solution-based mesh refinement to the LPM jet interaction configuration is more difficult because the flow is highly unsteady and there are many flow features of interest from weak interactions to strong. For example, the main bow shock is well resolved in Fig. 18, while the structures within the jet are not well-resolved. In axisymmetric computations, under-resolved areas can be easily picked on a graphical user interface to select additional refinement areas. However, this approach is sometimes hard to do in 3-D because of more complicated flow features. Moreover, manual intervention cannot be used in automating mesh refinement needed in unsteady flow computations, and 
manual intervention also increases the work of unstructured grid generation. Additional investigation on better flow feature detection sensors is needed and will be explored as part of future work. Additionally, implementing mesh coarsening can greatly improve mesh and solution quality without significant additional computation cost.

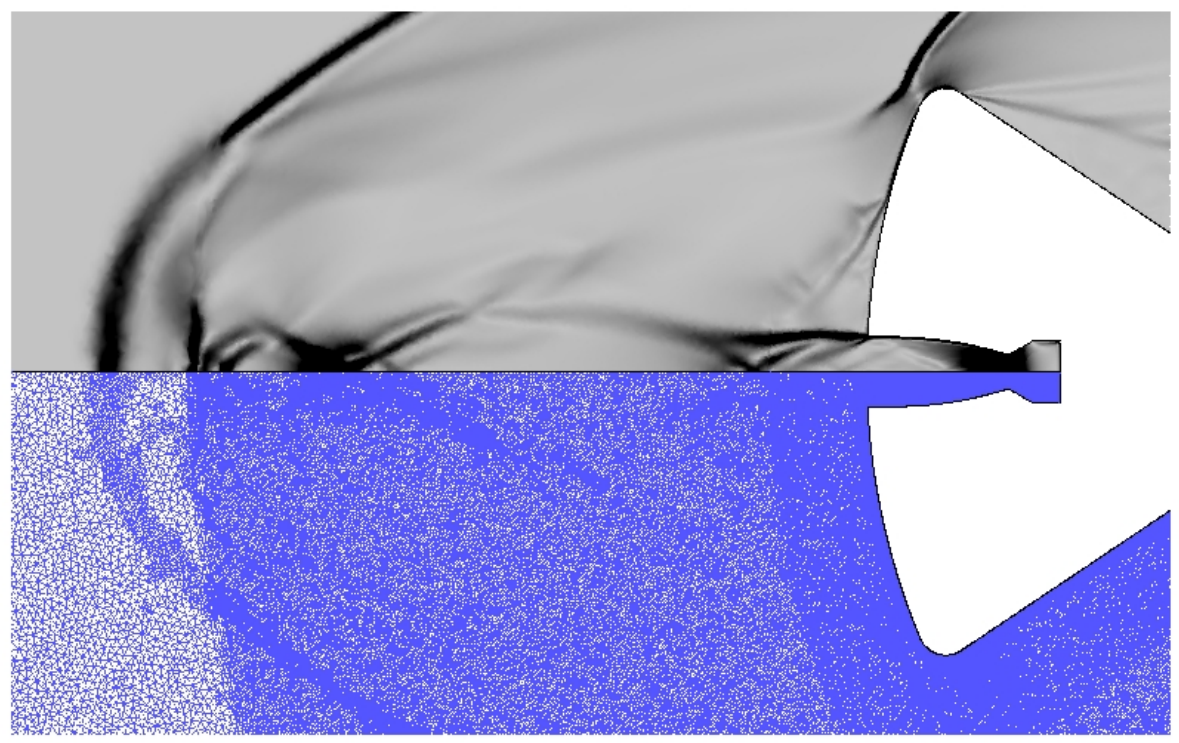

Fig. 18. Preliminary results of the solution-based mesh refinement method applied to the LPM jet.

\section{Conclusions}

The space-time CESE framework was used to numerically investigate the behavior of counterflowing jets ejected from a capsule against a supersonic freestream using unstructured triangular meshes. Special attention was given to understanding the unstable interaction known as the long penetration mode and how it transitions into the stable short penetration mode. The computed flow fields compare well with available experimental Schlieren images. However, the availability of unsteady flow data and flow contours with higher resolution helped obtain a more detailed understanding of the jet behavior including the cause of instability in the long penetration mode and how it transitions into a stable mode. Based on the present numerical investigation and knowledge gained from the literature, the source of instability of the counterflowing jet interaction was found to be closely related to the behavior of slightly under-expanded free jets. The diamond-shape shock cells from the under-expanded jets cause strong flow instability as the jet plume interacts with opposing freestream. It was observed that when the jet-pressure ratio of nozzle exit to pressure at the ambient outside the nozzle is large enough, the counterflowing jets behaved as highly under-expanded jets. Highly under-expanded jets end with a Mach disk termination and the overall flow is more stable. Based on the computational results, an increase in the jet flow rates or jet-to-freestream total pressure ratio transitions the overall flow from a stable detached bow shock for the case of no jet injection into a highly unstable long penetration mode, only to quickly revert back to the stable short penetration mode whose behavior is similar to that of a less-blunt capsule with no jet injection. The potential usage of solution-based mesh adaptation techniques was discussed to generate better meshes quickly and areas needing improvements, such as better flow-feature detection sensors, were identified. The outcome of this paper is closely related to other work in NASA's EDL project. More work will be carried out in the future for the retro-propulsion configurations related to the on-going wind tunnel experiments at NASA Langley Research Center.

\section{Acknowledgments}

Balaji Shankar Venkatachari and Gary Cheng acknowledge financial support by the Hypersonics Project under NASA's Fundamental Aeronautics Program (FAP) through cooperative agreement NNM07AA05A. Chau-Lyan Chang acknowledges support from NASA's Aerodynamics, Aerothermodynamics and Plasmadynamics (AAP) discipline of the Hypersonics project. The authors would also like to thank Deepak Bose of NASA Ames Research Center and Sin-Chung Chang of NASA Glenn Research Center for valuable technical input. This work was made possible in part by computing resources and technical support provided by the Alabama Supercomputer Authority. 


\section{References}

1 Korzun, A. M., Braun, R. D., and Cruz, J. R., "Survey of Supersonic Retropropulsion Technology for Mars Entry, Descent, and Landing," Journal of Spacecraft and Rockets, Vol. 46, No. 5, 2009, pp. 929-937.

2 Korzun, A. M., Cordell, Jr., C. E., and Braun, R. D., "Comparison of Inviscid and Viscous Aerodynamic Predictions of Supersonic Retropropulsion Flowfields," AIAA Paper 2010-5048, 2010.

3 Trumble, K. A., Schauerhamer, D. G., Kleb, W. L., Carlson, J-R., Buning, P. G., Edquist, K. T., and Barnhardt, M. D., “An Initial Assessment of Navier-Stokes Codes Applied to Supersonic Retro-Propulsion,” AIAA Paper 2010-5047, 2010.

4 Love, E. S., "The Effect of a Small Jet of Air Exhausting from the Nose of a Body of Revolution in Supersonic Flow," NACA RM L52I19a, Nov. 1952.

5 Moeckel, W. E., "Flow Separation Ahead of Blunt Bodies at Supersonic Speeds," NACA TN 2418, July 1951.

6 Huff, R. G., and Abdalla, K. L., "Mixing Characteristics Downstream of Core Region of High-Temperature Axisymmetric Jets Exhausting into Transonic and Supersonic Streams," NASA TM X-151, March 1960.

7 Stadler, J.R., and Inouye, M., “A Method of Reducing Heat Transfer to Blunt Bodies by Air Injection,” NACA RM A56B27a, May 1956.

8 Rashis, B., "Preliminary Indications of the Cooling Achieved by Injecting Water Upstream from the Stagnation Point of Hemispherical, $80^{\circ}$ Conical, and Flat-Faced Nose Shapes at a Stagnation Temperature of 4000F," NACA RM L57103, Oct. 1957.

9 Ferri, A., and Bloom, M. H., "Cooling by Jets Directed Upstream in Hypersonic Flow," Wright Air Development Center TN 56-382, Sept. 1957.

${ }^{10}$ Warren, C. H. E., “An Experimental Investigation of the Effect of Ejecting a Coolant Gas at the Nose of a Bluff Body," Journal of Fluid Mechanics, Vol. 8, Pt. 3, 1960, pp. 400-417.

${ }^{11}$ Finley, P. J., "The Flow of a Jet from a Body Opposing a Supersonic Free Stream,” Journal of Fluid Mechanics, Vol. 26, Pt. 2, 1966, pp. 337-368.

12 Bushnell, D. M., and Huffman, J. K., "Forward Penetration of Liquid Water and Liquid Nitrogen from the Orifice at the Stagnation Point of a Hemispherically Blunted Body in Hypersonic Flow," NASA TM X- 1493, March 1968.

13 Jarvinen, P. O., and Adams, R. H., "The Effects of Retrorockets on the Aerodynamic Characteristics of Conical Aeroshell Planetary Entry Vehicles," AIAA Paper 70-219, 1970.

${ }^{14}$ McGhee, R. J., "Effects of a Retronozzle Located at the Apex of a $140^{\circ}$ Blunt Cone at Mach Numbers of 3.00, 4.50, and 6.00," NASA TN D- 6002, Jan. 1971.

${ }^{15}$ Daso, E. O., Beaulieu, W., and Hager, J. O., "Prediction of Drag Reduction in Supersonic and Hypersonic Flows with Counter-Flow Jets," AIAA Paper 2002-5115, 2002.

${ }^{16}$ Daso, E. O., Pritchett, V. E., Wang, T. S., Ota, D. K., Blankson, I. M., and Auslender. A. H., “The Dynamics of Shock Dispersion and Interactions in Supersonic Freestreams with Counterflowing Jets," AIAA Journal, Vol. 47, No. 6, 2009, pp. 1313-1326.

${ }^{17}$ Chang, S. C., "The Method of Space-Time Conservation Element and Solution Element - A New Approach for Solving the NavierStokes and Euler Equations," Journal of Computational Physics, Vol. 119, 1995, pp. 295-324.

${ }^{18}$ Chang, S. C., Wang, X. Y., and To, W. M., "Application of the Space-Time Conservation Element and Solution Element Method to One-Dimensional Convection-Diffusion Problems," Journal of Computational Physics, Vol. 165, 2000, pp. 189-215.

${ }^{19}$ Kim, C. K., Yu, S. T., and Zhang, Z. C., "Cavity Flow in Scramjet Engine by the Space-Time Conservation Element and Solution Element Method," AIAA Journal, Vol. 42, No. 5, 2004, pp. 912- 919.

${ }^{20}$ Qin, J., Yu, S. T., Zhang, Z. C., and Lai, M. C., "Direct Calculations of Cavitating Flows in Fuel Delivery Pipe by the Space-Time CESE Method," Journal of Fuels and Lubricants, SAE Transactions, Vol. 108, No. 4, 2000, pp. 1720-1725.

${ }^{21}$ Zhang, M., Yu, S. T., Lin, S. C., Chang, S. C., and Blankson, I., "Solving Magnetohydrodynamic Equations Without Special Treatment for Divergence-Free Magnetic Field," AIAA Journal, Vol. 42, No. 12, 2004, pp. 2605-2608.

${ }^{22}$ Chang, S. C., and Wang, X. Y., "Multi-Dimensional Courant Number Insensitive CESE Euler Solvers for Application Involving Highly Non-uniform Meshes," AIAA Paper 2003-5285, 2003.

${ }^{23}$ Chang, C-L., Venkatachari, B. S., and Cheng, G. C., "Effect of Counterflow Jet on a Supersonic Reentry Capsule," AIAA Paper 20064776, 2006

${ }^{24}$ Chang, C-L., "Three-Dimensional Navier-Stokes Calculations Using the Modified Space-Time CESE Method," AIAA Paper 20075818, 2007.

${ }^{25}$ Venkatachari, B., Cheng, G. C., Soni, B. K., and Chang, S. C., "Validation and Verification of Courant Number Insensitive CE/SE Method for Transient Viscous Flow Simulations," Mathematics and Computers in Simulation, Vol. 78, Issue 5-6, 2008, pp 653-670.

${ }^{26}$ Cheng, G. C., Venkatachari, B. S., Chang, C.-L., and Chang, S. C., "Comparative Study of Different Numerical Approaches in SpaceTime CESE Framework for High-fidelity Flow Simulations," Computers and Fluids, Vol. 45, No.1, 2011, pp 47-54.

${ }^{27}$ Peery, K. M., and Imlay, S. T., "Blunt-body flow simulation," AIAA Paper 88-2904, 1988.

${ }^{28}$ Ito, Y. and Nakahashi, K., "Direct Surface Triangulation Using Stereolithography Data," AIAA Journal, Vol. 40, No.3, 2002, pp. 490496.

${ }^{29}$ Ito, Y., Shih, A. M., Koomullil, R. P., Kasmai, N., Jankun-Kelly, M. and Thompson, D., "Solution Adaptive Mesh Generation Using Feature-Aligned Embedded Surface Meshes," AIAA Journal, Vol. 47, No.8, 2009, pp. 1879-1888.

${ }^{30}$ Cheng, G. C., Neroorkar, K. D., Chen, Y-S., Wang, T-S., and Daso, E. O., "Numerical Study of Flow Augmented Thermal Management for Entry and Re-entry Environments," AIAA Paper 2007-4560, 2007.

${ }^{31}$ Romeo, D. and Sterrett, J., "Exploratory Investigation of the Effect of a Forward-Facing Jet on the Bow Shock of a Blunt Body in a Mach Number 6 Free Stream," NASA TN D-1605, February 1963.

${ }^{32}$ Lovely, D., and Haimes, R., "Shock Detection from Computational Fluid Dynamics Results," AIAA Paper 1999-3285, 1999.

${ }^{33}$ Soni, B. K., Koomullil, R., Thompson, D. S., and Thornburg, H., "Solution Adaptive Grid Strategies Based on Point Redistribution," Computer Methods in Applied Mechanics and Engineering, Vol. 189, Issue 4, 2000, pp. 1183-1204. 